\title{
Hom-Lie Algebras and Hom-Lie Groups, Integration and Differentiation
}

\author{
Jun JIANG ${ }^{\dagger}$, Satyendra Kumar MISHRA ${ }^{\ddagger}$ and Yunhe SHENG ${ }^{\dagger}$ \\ $\dagger$ Department of Mathematics, Jilin University, Changchun, Jilin Province, 130012, China \\ E-mail: jiangjun20@mails.jlu.edu.cn, shengyh@jlu.edu.cn \\ $\ddagger$ Statistics and Mathematics Unit, Indian Statistical Institute Bangalore, India \\ E-mail: satyamsr10@gmail.com
}

Received June 01, 2020, in final form December 10, 2020; Published online December 17, 2020

https://doi.org/10.3842/SIGMA.2020.137

\begin{abstract}
In this paper, we introduce the notion of a (regular) Hom-Lie group. We associate a Hom-Lie algebra to a Hom-Lie group and show that every regular Hom-Lie algebra is integrable. Then, we define a Hom-exponential (Hexp) map from the Hom-Lie algebra of a Hom-Lie group to the Hom-Lie group and discuss the universality of this Hexp map. We also describe a Hom-Lie group action on a smooth manifold. Subsequently, we give the notion of an adjoint representation of a Hom-Lie group on its Hom-Lie algebra. At last, we integrate the Hom-Lie algebra $(\mathfrak{g l}(V),[\cdot, \cdot], \mathrm{Ad})$, and the derivation Hom-Lie algebra of a Hom-Lie algebra.
\end{abstract}

Key words: Hom-Lie algebra; Hom-Lie group; derivation; automorphism; integration

2020 Mathematics Subject Classification: 17B40; 17B61; 22E60; 58A32

\section{Introduction}

The notion of a Hom-Lie algebra first appeared in the study of quantum deformations of Witt and Virasoro algebras in [6]. Hom-Lie algebras are generalizations of Lie algebras, where the Jacobi identity is twisted by a linear map, called the Hom-Jacobi identity. It is known that qdeformations of the Witt and the Virasoro algebras have the structure of a Hom-Lie algebra [6, 9]. There is a growing interest in Hom-algebraic structures because of their close relationship with the discrete and deformed vector fields, and differential calculus [6, 10, 11]. In particular, representations and deformations of Hom-Lie algebras were studied in [1, 15, 17]; a categorical interpretation of Hom-Lie algebras was described in [3]; the categorification of Hom-Lie algebras was given in [18]; geometric and algebraic generalizations of Hom-Lie algebras were given in [4, 14, 16]; quantization of Hom-Lie algebras was studied in [21]; and the universal enveloping algebra of Hom-Lie algebras was studied in [13, 20].

The notion of Hom-groups was initially introduced by Caenepeel and Goyvaerts in [3]. In [13], Laurent-Gengoux, Makhlouf and Teles first gave a new construction of the universal enveloping algebra that is different from the one in [20]. This new construction leads to a Hom-Hopf algebra structure on the universal enveloping algebra of a Hom-Lie algebra. Moreover, one can associate a Hom-group to any Hom-Lie algebra by considering group-like elements in its universal enveloping algebra. Recently, M. Hassanzadeh developed representations and a (co)homology theory for Hom-groups in [7]. He also proved Lagrange's theorem for finite Hom-groups in [8]. The recent developments on Hom-groups (see [7, 8, 13]) make it natural to study Hom-Lie groups and to explore the relationship between Hom-Lie groups and Hom-Lie algebras.

In this paper, we introduce a (real) Hom-Lie group as a Hom-group $\left(G, \diamond, e_{\Phi}, \Phi\right)$, where the underlying set $G$ is a (real) smooth manifold, the Hom-group operations (such as the product and 
the inverse) are smooth maps, and the underlying structure map $\Phi: G \rightarrow G$ is a diffeomorphism. We associate a Hom-Lie algebra to a Hom-Lie group by considering the notion of left-invariant sections of the pullback bundle $\Phi ! T G$. We define one-parameter Hom-Lie subgroups of a HomLie group and discuss a Hom-analogue of the exponential map from the Hom-Lie algebra of a Hom-Lie group to the Hom-Lie group. Later on, we consider Hom-Lie group actions on a manifold $M$ with respect to a map $\iota \in \operatorname{Diff}(M)$ and define an adjoint representation of a HomLie group on its Hom-Lie algebra. Finally, we discuss the integration of the Hom-Lie algebra $\left(\mathfrak{g l}(V),[\cdot, \cdot]_{\beta}, \operatorname{Ad}_{\beta}\right)$ and the derivation Hom-Lie algebra $\operatorname{Der}(\mathfrak{g})$ of a Hom-Lie algebra $\left(\mathfrak{g},[\cdot, \cdot]_{\mathfrak{g}}, \phi_{\mathfrak{g}}\right)$.

All the results in the paper are under the regularity hypothesis. As appeared in the literature, e.g., [12], Hom-Lie algebras are not necessarily regular. We will explore this more general case in the future.

The paper is organized as follows. In Section 2, we recall some basic definitions and results concerning Hom-Lie algebras and Hom-groups. In Section 3, we define the notion of a HomLie group with some useful examples. If $\left(G, \diamond, e_{\Phi}, \Phi\right)$ is a Hom-Lie group, then we show that the space of left-invariant sections of the pullback bundle $\Phi^{!} T G$ has a Hom-Lie algebra structure. Consequently, we deduce a Hom-Lie algebra structure on the fibre of the pullback bundle $\Phi^{!} T G$ at $e_{\Phi}$. In this way, we associate a Hom-Lie algebra $\left(\mathfrak{g}^{!},[\cdot, \cdot]_{\mathfrak{g}^{!}}, \phi_{\mathfrak{g} !}\right)$ to the Hom-Lie group $\left(G, \diamond, e_{\Phi}, \Phi\right)$, where $\mathfrak{g}^{!}:=\Phi^{!} T_{e_{\Phi}} G$. We also show that every regular Hom-Lie algebra is integrable. Next, we define one-parameter Hom-Lie subgroups of a Hom-Lie group $\left(G, \diamond, e_{\Phi}, \Phi\right)$ in terms of a weak homomorphism of Hom-Lie groups. We establish a one-to-one correspondence

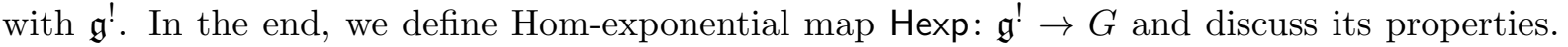
In Section 4, we study Hom-Lie group actions on a smooth manifold $M$ with respect to a diffeomorphism $\iota \in \operatorname{Diff}(M)$. We define representations of a Hom-Lie group on a vector space $V$ with respect to a map $\beta \in \mathrm{GL}(V)$, which leads to the notion of an adjoint representation of a Hom-Lie group on the associated Hom-Lie algebra. In Section 5, we consider the Hom-Lie $\operatorname{group}\left(\mathrm{GL}(V), \diamond, \beta, \operatorname{Ad}_{\beta}\right)$, where $V$ is a vector space and $\beta \in \mathrm{GL}(V)$. We show that the triple $\left(\mathfrak{g l}(V),[\cdot, \cdot]_{\beta}, \operatorname{Ad}_{\beta}\right)$ is the Hom-Lie algebra of the Hom-Lie group $\left(\operatorname{GL}(V), \diamond, \beta, \operatorname{Ad}_{\beta}\right)$. In the last section, we show that the derivation Hom-Lie algebra $\operatorname{Der}(\mathfrak{g})$ is the Hom-Lie algebra of the Hom-Lie group of automorphisms of $\left(\mathfrak{g},[\cdot, \cdot]_{\mathfrak{g}}, \phi_{\mathfrak{g}}\right)$.

\section{Preliminaries}

In this section, we first recall definitions of Hom-Lie algebras and Hom-groups.

\subsection{Hom-Lie algebras}

Definition 2.1. A (multiplicative) Hom-Lie algebra is a triple $\left(\mathfrak{g},[\cdot, \cdot]_{\mathfrak{g}}, \phi_{\mathfrak{g}}\right)$ consisting of a vector space $\mathfrak{g}$, a skew-symmetric bilinear map (bracket) $[\cdot, \cdot]_{\mathfrak{g}}: \wedge^{2} \mathfrak{g} \longrightarrow \mathfrak{g}$, and a linear map $\phi_{\mathfrak{g}}: \mathfrak{g} \rightarrow \mathfrak{g}$ preserving the bracket, such that the following Hom-Jacobi identity with respect to $\phi_{\mathfrak{g}}$ is satisfied:

$$
\left[\phi_{\mathfrak{g}}(x),[y, z]_{\mathfrak{g}}\right]_{\mathfrak{g}}+\left[\phi_{\mathfrak{g}}(y),[z, x]_{\mathfrak{g}}\right]_{\mathfrak{g}}+\left[\phi_{\mathfrak{g}}(z),[x, y]_{\mathfrak{g}}\right]_{\mathfrak{g}}=0, \quad \forall x, y, z \in \mathfrak{g}
$$

A Hom-Lie algebra $\left(\mathfrak{g},[\cdot, \cdot]_{\mathfrak{g}}, \phi_{\mathfrak{g}}\right)$ is called a regular Hom-Lie algebra if $\phi_{\mathfrak{g}}$ is an invertible map.

Lemma 2.2. Let $\left(\mathfrak{g},[\cdot, \cdot]_{\mathfrak{g}}, \phi_{\mathfrak{g}}\right)$ be a regular Hom-Lie algebra. Then $\left(\mathfrak{g},[\cdot, \cdot]_{\text {Lie }}\right)$ is a Lie algebra, where the Lie bracket $[\cdot, \cdot]_{\text {Lie }}$ is given by $[x, y]_{\text {Lie }}=\left[\phi_{\mathfrak{g}}^{-1}(x), \phi_{\mathfrak{g}}^{-1}(y)\right]_{\mathfrak{g}}$ for all $x, y \in \mathfrak{g}$.

In the sequel, we always assume that $\phi_{\mathfrak{g}}$ is an invertible map. That is, in this paper, all the Hom-Lie algebras are assumed to be regular Hom-Lie algebras.

Remark 2.3. In [3], the authors used a monoidal categorical approach to give an intrinsic study of regular Hom-type algebraic structures. In particular, a regular Hom-Lie algebra is 
called a monoidal Hom-Lie algebra there. See [2] for the categorical framework study of the BiHom-type structures.

Definition 2.4. Let $\left(\mathfrak{g},[\cdot, \cdot]_{\mathfrak{g}}, \phi_{\mathfrak{g}}\right)$ and $\left(\mathfrak{h},[\cdot, \cdot]_{\mathfrak{h}}, \phi_{\mathfrak{h}}\right)$ be Hom-Lie algebras.

(i) A linear map $f: \mathfrak{g} \rightarrow \mathfrak{h}$ is called a weak homomorphism of Hom-Lie algebras if

$$
\phi_{\mathfrak{h}} f[x, y]_{\mathfrak{g}}=\left[f\left(\phi_{\mathfrak{g}}(x)\right), f\left(\phi_{\mathfrak{g}}(y)\right)\right]_{\mathfrak{h}}, \quad \forall x, y \in \mathfrak{g} .
$$

(ii) A weak homomorphism $f: \mathfrak{g} \rightarrow \mathfrak{h}$ is called a homomorphism if $f$ also satisfies

$$
f \circ \phi_{\mathfrak{g}}=\phi_{\mathfrak{h}} \circ f .
$$

Definition 2.5. A representation of a Hom-Lie algebra $\left(\mathfrak{g},[\cdot, \cdot]_{\mathfrak{g}}, \phi_{\mathfrak{g}}\right)$ on a vector space $V$ with respect to $\beta \in \mathfrak{g l}(V)$ is a linear map $\rho: \mathfrak{g} \rightarrow \mathfrak{g l}(V)$ such that for all $x, y \in \mathfrak{g}$, the following equations are satisfied

$$
\begin{aligned}
& \rho\left(\phi_{\mathfrak{g}}(x)\right) \circ \beta=\beta \circ \rho(x), \\
& \rho\left([x, y]_{\mathfrak{g}}\right) \circ \beta=\rho\left(\phi_{\mathfrak{g}}(x)\right) \circ \rho(y)-\rho\left(\phi_{\mathfrak{g}}(y)\right) \circ \rho(x) .
\end{aligned}
$$

For all $x \in \mathfrak{g}$, let us define a map ad $\operatorname{ad}_{x}: \mathfrak{g} \rightarrow \mathfrak{g}$ by

$$
\operatorname{ad}_{x}(y)=[x, y]_{\mathfrak{g}}, \quad \forall y \in \mathfrak{g} .
$$

Then ad: $\mathfrak{g} \longrightarrow \mathfrak{g l}(\mathfrak{g})$ is a representation of the Hom-Lie algebra $\left(\mathfrak{g},[\cdot, \cdot]_{\mathfrak{g}}, \phi_{\mathfrak{g}}\right)$ on $\mathfrak{g}$ with respect to $\phi_{\mathfrak{g}}$, which is called the adjoint representation.

Let $(\rho, V, \beta)$ be a representation of a Hom-Lie algebra $\left(\mathfrak{g},[\cdot, \cdot]_{\mathfrak{g}}, \phi_{\mathfrak{g}}\right)$ on a vector space $V$ with respect to the map $\beta \in \mathfrak{g l}(V)$. Then let us recall from [5] that the cohomology of the HomLie algebra $\left(\mathfrak{g},[\cdot, \cdot]_{\mathfrak{g}}, \phi_{\mathfrak{g}}\right)$ with coefficients in $(\rho, V, \beta)$ is the cohomology of the cochain complex $C^{k}(\mathfrak{g}, V)=\operatorname{Hom}\left(\wedge^{k} \mathfrak{g}, V\right)$ with the coboundary operator $d: C^{k}(\mathfrak{g}, V) \rightarrow C^{k+1}(\mathfrak{g}, V)$ defined by

$$
\begin{aligned}
(d f) & \left(x_{1}, \ldots, x_{k+1}\right)=\sum_{i=1}^{k+1}(-1)^{i+1} \rho\left(x_{i}\right)\left(f\left(\phi_{\mathfrak{g}}^{-1} x_{1}, \ldots, \widehat{\phi_{\mathfrak{g}}^{-1} x_{i}}, \ldots, \phi_{\mathfrak{g}}^{-1} x_{k+1}\right)\right) \\
& +\sum_{i<j}(-1)^{i+j} \beta f\left(\left[\phi_{\mathfrak{g}}^{-2} x_{i}, \phi_{\mathfrak{g}}^{-2} x_{j}\right]_{\mathfrak{g}}, \phi_{\mathfrak{g}}^{-1} x_{1}, \ldots, \widehat{\phi_{\mathfrak{g}}^{-1} x_{i}}, \ldots, \widehat{\phi_{\mathfrak{g}}^{-1} x_{j}}, \ldots, \phi_{\mathfrak{g}}^{-1} x_{k+1}\right) .
\end{aligned}
$$

The fact that $d^{2}=0$ is proved in [5]. Denote by $\mathcal{Z}^{k}(\mathfrak{g} ; \rho)$ and $\mathcal{B}^{k}(\mathfrak{g} ; \rho)$ the sets of $k$-cocycles and $k$ coboundaries respectively. We define the $k$-th cohomology group $\mathcal{H}^{k}(\mathfrak{g} ; \rho)$ to be $\mathcal{Z}^{k}(\mathfrak{g} ; \rho) / \mathcal{B}^{k}(\mathfrak{g} ; \rho)$.

Let $\left(\mathrm{ad}, \mathfrak{g}, \phi_{\mathfrak{g}}\right)$ be the adjoint representation. For any 0 -Hom-cochain $x \in \mathfrak{g}=C^{0}(\mathfrak{g}, \mathfrak{g})$, we have

$$
(d x)(y)=[y, x]_{\mathfrak{g}}, \quad \forall y \in \mathfrak{g} .
$$

Thus, we have $d x=0$ if and only if $x \in \operatorname{Cen}(\mathfrak{g})$, where $\operatorname{Cen}(\mathfrak{g})$ denotes the center of $\mathfrak{g}$. Therefore,

$$
\mathcal{H}^{0}(\mathfrak{g}, \text { ad })=\mathcal{Z}^{0}(\mathfrak{g}, \text { ad })=\operatorname{Cen}(\mathfrak{g}) .
$$

Definition 2.6. A linear map $D: \mathfrak{g} \rightarrow \mathfrak{g}$ is called a derivation of a Hom-Lie algebra $\left(\mathfrak{g},[\cdot, \cdot]_{\mathfrak{g}}, \phi_{\mathfrak{g}}\right)$ if the following identity holds:

$$
D[x, y]_{\mathfrak{g}}=\left[\phi(x),\left(\operatorname{Ad}_{\phi_{\mathfrak{g}}^{-1}} D\right)(y)\right]_{\mathfrak{g}}+\left[\left(\operatorname{Ad}_{\phi_{\mathfrak{g}}^{-1}} D\right)(x), \phi(y)\right]_{\mathfrak{g}}, \quad \forall x, y \in \mathfrak{g} .
$$

We denote the space of derivations of the Hom-Lie algebra $\left(\mathfrak{g},[\cdot, \cdot]_{\mathfrak{g}}, \phi_{\mathfrak{g}}\right)$ by $\operatorname{Der}(\mathfrak{g})$. 
Let us observe that if $D \circ \phi=\phi \circ D$ in the Definition 2.6, then any derivation of the Hom-Lie algebra $\left(\mathfrak{g},[\cdot, \cdot]_{\mathfrak{g}}, \phi_{\mathfrak{g}}\right)$ is a $\phi_{\mathfrak{g}}$-derivation (see [17] for more details).

Example 2.7. Let $\left(\mathfrak{g},[\cdot, \cdot]_{\mathfrak{g}}, \phi_{\mathfrak{g}}\right)$ be a Hom-Lie algebra. For each $x \in \mathfrak{g}, \operatorname{ad}_{x}$ is a derivation of $\left(\mathfrak{g},[\cdot, \cdot]_{\mathfrak{g}}, \phi_{\mathfrak{g}}\right)$ that we call an "inner derivation".

Let us denote the space of inner derivations by $\operatorname{lnn} \operatorname{Der}(\mathfrak{g})$. It is immediate to see that

$$
\mathcal{Z}^{1}(\mathfrak{g}, \mathrm{ad})=\operatorname{Der}(\mathfrak{g}), \quad \mathcal{B}^{1}(\mathfrak{g}, \mathrm{ad})=\operatorname{lnn} \operatorname{Der}(\mathfrak{g}) .
$$

Therefore,

$$
\mathcal{H}^{1}(\mathfrak{g}, \mathrm{ad})=\operatorname{Der}(\mathfrak{g}) / \operatorname{InnDer}(\mathfrak{g})=\operatorname{OutDer}(\mathfrak{g}) .
$$

Here, OutDer $(\mathfrak{g})$ denotes the space of outer derivations of the Hom-Lie algebra $\left(\mathfrak{g},[\cdot, \cdot]_{\mathfrak{g}}, \phi_{\mathfrak{g}}\right)$.

Let $V$ be a vector space, and $\beta \in \mathrm{GL}(V)$. Let us define a skew-symmetric bilinear bracket operation $[\cdot, \cdot]_{\beta}: \wedge^{2} \mathfrak{g l}(V) \longrightarrow \mathfrak{g l}(V)$ by

$$
[A, B]_{\beta}=\beta \circ A \circ \beta^{-1} \circ B \circ \beta^{-1}-\beta \circ B \circ \beta^{-1} \circ A \circ \beta^{-1}, \quad \forall A, B \in \mathfrak{g l}(V) .
$$

We also define a map $\operatorname{Ad}_{\beta}: \mathfrak{g l}(V) \rightarrow \mathfrak{g l}(V)$ by

$$
\operatorname{Ad}_{\beta}(A)=\beta \circ A \circ \beta^{-1}, \quad \forall A \in \mathfrak{g l}(V) .
$$

With the above notations, we have the following proposition.

Proposition 2.8 ([19, Proposition 4.1]). The triple $\left(\mathfrak{g l}(V),[\cdot, \cdot]_{\beta}, \mathrm{Ad}_{\beta}\right)$ is a regular Hom-Lie algebra.

The Hom-Lie algebra $\left(\mathfrak{g l}(V),[\cdot, \cdot]_{\beta}, \mathrm{Ad}_{\beta}\right)$ plays an important role in the representation theory of Hom-Lie algebras. See [19] for more details.

\subsection{Hom-groups}

Throughout this paper, we consider regular Hom-groups that is the case when the structure map is invertible, and this notion can be traced back to Caenepeel and Goyvaerts's pioneering work [3]. The axioms in the following definition of Hom-group is different from the one in $[7,8,13]$. However, we show that if the structure map is invertible, then some axioms in the original definition are redundant and can be obtained from the Hom-associativity condition.

Definition 2.9. A (regular) Hom-group is a set $G$ equipped with a product $\diamond: G \times G \longrightarrow G$, a bijective map $\Phi: G \longrightarrow G$ such that the following axioms are satisfied

(i) $\Phi(x \diamond y)=\Phi(x) \diamond \Phi(y)$;

(ii) the product is Hom-associative, i.e.,

$$
\Phi(x) \diamond(y \diamond z)=(x \diamond y) \diamond \Phi(z), \quad \forall x, y, z \in G ;
$$

(iii) there exists a unique Hom-unit $e_{\Phi} \in G$ such that

$$
x \diamond e_{\Phi}=e_{\Phi} \diamond x=\Phi(x), \quad \forall x \in G ;
$$

(iv) for each $x \in G$, there exists an element $x^{-1} \in G$ satisfying the following condition

$$
x \diamond x^{-1}=x^{-1} \diamond x=e_{\Phi} .
$$

We denote a Hom-group by $\left(G, \diamond, e_{\Phi}, \Phi\right)$. 
Remark 2.10. The category of sets (Sets, $\times,\{*\}, \tau$ ) (where $\tau$ is the twist) is a symmetric (strict) monoidal category. Algebras (monoids) in Sets are also bialgebras since every set $X$ has a unique structure of coalgebra in Sets, namely $\Delta(x)=(x, x)$ and $\varepsilon(x)=*$, for all $x \in X$. A Hopf algebra in Sets is a group. Let $X$ be a set and $\pi$ be a permutation. Then $(X, \pi, \Delta, \varepsilon)$ is a Homcomonoid, where $\Delta: X \rightarrow X \times X$ and $\varepsilon: X \rightarrow X$ are the maps given by $\Delta(x)=\left(\pi^{-1}(x), \pi^{-1}(x)\right)$ and $\varepsilon(x)=*$. Similarly, if $\phi$ is an automorphism of a group $G$, then $(G, \phi)$ with structure maps

$$
g \cdot h=\phi(g h), \quad \eta(*)=1_{G}, \quad \varepsilon(g)=*, \quad \Delta(g)=\left(\phi^{-1}(g), \phi^{-1}(g)\right), \quad S(g)=g^{-1},
$$

is a Hom-group, that is a Hopf algebra in $\tilde{\mathcal{H}}$ (Sets) in [3, Section 5]. Thus a monoidal categorical approach can give an intrinsic study of regular Hom-groups.

Remark 2.11. Note that the definition of a Hom-group in [8] consists of the axiom $\Phi\left(e_{\Phi}\right)=e_{\Phi}$. In Proposition 2.13, we show that this axiom is redundant in the regular case. Let us recall the Hom-invertibility condition in the definition of a Hom-group $(G, \Phi)$ in [13]: for each $x \in G$, there exists a positive integer $k$ such that

$$
\Phi^{k}\left(x \diamond x^{-1}\right)=\Phi^{k}\left(x^{-1} \diamond x\right)=e_{\Phi},
$$

and the smallest such integer $k$ is called the invertibility index of $x \in G$. In the regular case, it is immediate to see that the Hom-invertibility condition is equivalent to the condition (iv) in Definition 2.9 .

Example 2.12. Let $(G, \mu, e)$ be a group and $\phi: G \rightarrow G$ be a group automorphism. Then the tuple $\left(G, \mu_{\phi}, e, \phi\right)$ with the product $\mu_{\phi}=\phi \circ \mu$, is a Hom-group. In particular, the tuple $(\mathbb{R},+, 0, \mathrm{Id})$ is a Hom-group, which will be used in our later definition of one-parameter Hom-Lie subgroups.

It is straightforward to obtain the following properties, which were also given in [3].

Proposition 2.13. Let $\left(G, \diamond, e_{\Phi}, \Phi\right)$ be a Hom-group. Then we have the following properties.

(i) $\Phi\left(e_{\Phi}\right)=e_{\Phi}$;

(ii) for each $x \in G$, there exists a unique inverse $x_{\Phi}^{-1}$ such that

$$
x \diamond x_{\Phi}^{-1}=x_{\Phi}^{-1} \diamond x=e_{\Phi}
$$

(iii) $(x \diamond y)^{-1}=y^{-1} \diamond x^{-1}, \forall x, y \in G$.

Definition 2.14. Let $\left(G, \diamond_{G}, e_{\Phi}, \Phi\right)$ and $\left(H, \diamond_{H}, e_{\Psi}, \Psi\right)$ be two Hom-groups.

(a) A homomorphism of Hom-groups is a map $f: G \rightarrow H$ such that $f\left(e_{\Phi}\right)=e_{\Psi}$ and $f\left(x \diamond_{G} y\right)=$ $f(x) \diamond_{H} f(y)$ for all $x, y \in G$.

(b) A weak homomorphism of Hom-groups is a map $f: G \rightarrow H$ such that $f\left(e_{\Phi}\right)=e_{\Psi}$ and $\Psi \circ f\left(x \diamond_{G} y\right)=(f \circ \Phi(x)) \diamond_{H}(f \circ \Phi(y))$ for all $x, y \in G$.

Let us observe that for a homomorphism $f:\left(G, \diamond_{G}, e_{\Phi}, \Phi\right) \rightarrow\left(H, \diamond_{H}, e_{\Psi}, \Psi\right)$, the commutativity condition: $\Psi \circ f=f \circ \Phi$ holds. It follows by the definition of a homomorphism and the identities: $\Phi\left(e_{\Phi}\right)=e_{\Phi}$, and $\Psi\left(e_{\Psi}\right)=e_{\Psi}$. Furthermore, any homomorphism of Hom-groups is also a weak homomorphism, however, the converse may not be true.

\section{Hom-Lie groups and Hom-Lie algebras}

Let $\mathbb{R}$ be the field of real numbers. From here onwards, we consider all manifolds, vector spaces over the field $\mathbb{R}$, and all the linear maps are considered to be $\mathbb{R}$-linear unless otherwise stated. 


\subsection{Hom-Lie groups}

Definition 3.1. A real Hom-Lie group is a Hom-group $\left(G, \diamond, e_{\Phi}, \Phi\right)$, in which $G$ is also a smooth real manifold, the map $\Phi: G \rightarrow G$ is a diffeomorphism, and the Hom-group operations (product and inversion) are smooth maps with respect to the topology of $G$.

Example 3.2. Let $(G, \cdot)$ be a Lie group with identity $e$ and $\Phi:(G, \cdot) \rightarrow(G, \cdot)$ be an automorphism. Then the tuple $\left(G, \diamond, e_{\Phi}=e, \Phi\right)$ is a Hom-Lie group, where the product $\diamond$ is defined by

$$
a \diamond b=\Phi(a) \cdot \Phi(b), \quad \forall a, b \in G .
$$

Let $V$ be a vector space and $\beta \in \mathrm{GL}(V)$. Then let us define a product $\diamond: \operatorname{GL}(V) \times \mathrm{GL}(V) \longrightarrow$ $\mathrm{GL}(V)$ by

$$
A \diamond B=\beta \circ A \circ \beta^{-1} \circ B \circ \beta^{-1}, \quad \forall A, B \in \mathrm{GL}(V) .
$$

Proposition 3.3. The tuple $\left(\mathrm{GL}(V), \diamond, \beta, \mathrm{Ad}_{\beta}\right)$ is a Hom-Lie group, where the product $\diamond$ is given by (3.1), the Hom-unit is $\beta$, and the map $\operatorname{Ad}_{\beta}: \mathrm{GL}(V) \rightarrow \mathrm{GL}(V)$ is defined by

$$
\operatorname{Ad}_{\beta}(A)=\beta \circ A \circ \beta^{-1}, \quad \forall A \in \mathrm{GL}(V) .
$$

Proof. For all $A, B \in \mathrm{GL}(V)$, we have

$$
\begin{aligned}
\operatorname{Ad}_{\beta}(A \diamond B) & =\operatorname{Ad}_{\beta}\left(\beta \circ A \circ \beta^{-1} \circ B \circ \beta^{-1}\right)=\beta^{2} \circ A \circ \beta^{-1} \circ B \circ \beta^{-2} \\
& =\beta \circ \operatorname{Ad}_{\beta}(A) \circ \beta^{-1} \circ \operatorname{Ad}_{\beta}(B) \circ \beta^{-1}=\operatorname{Ad}_{\beta}(A) \diamond \operatorname{Ad}_{\beta}(B) .
\end{aligned}
$$

Thus, condition (i) in Definition 2.9 holds. For all $A, B, C \in \mathrm{GL}(V)$, it easily follows that

$$
\begin{aligned}
(A \diamond B) \diamond\left(\operatorname{Ad}_{\beta}(C)\right) & =\left(\beta \circ A \circ \beta^{-1} \circ B \circ \beta^{-1}\right) \diamond\left(\beta \circ C \circ \beta^{-1}\right) \\
& =\beta^{2} \circ A \circ \beta^{-1} \circ B \circ \beta^{-1} \circ C \circ \beta^{-2} \\
& =\beta \circ \operatorname{Ad}_{\beta}(A) \circ \beta^{-1} \circ(B \diamond C) \circ \beta^{-1} \\
& =\operatorname{Ad}_{\beta}(A) \diamond(B \diamond C),
\end{aligned}
$$

which implies that the product $\diamond$ is Hom-associative. Next, we have

$$
A \diamond \beta=\beta \circ A \circ \beta^{-1} \circ \beta \circ \beta^{-1}=\operatorname{Ad}_{\beta}(A), \quad \forall A \in \mathrm{GL}(V) .
$$

Similarly,

$$
\beta \diamond A=\operatorname{Ad}_{\beta}(A), \quad \forall A \in \mathrm{GL}(V) .
$$

Therefore, $\beta$ is the Hom-unit. Finally, we have the following expression

$$
A \diamond\left(\beta \circ A^{-1} \circ \beta\right)=\beta \circ A \circ \beta^{-1} \circ\left(\beta \circ A^{-1} \circ \beta\right) \circ \beta^{-1}=\beta=\left(\beta \circ A^{-1} \circ \beta\right) \diamond A,
$$

for any $A \in \mathrm{GL}(V)$, i.e., $\beta \circ A^{-1} \circ \beta$ is the Hom-inverse of $A$. Hence, the tuple $\left(\operatorname{GL}(V), \diamond, \beta, \operatorname{Ad}_{\beta}\right)$ is a Hom-group.

Example 3.4. Let $M$ be a smooth manifold. Let us denote by $\operatorname{Diff}(M)$, the set of diffeomorphisms of $M$. If $\iota \in \operatorname{Diff}(M)$, then the tuple ( $\left.\operatorname{Diff}(M), \diamond, \iota, \operatorname{Ad}_{\iota}\right)$ is a Hom-Lie group, where

(i) the product $\diamond$ is given by the following equation:

$$
f \diamond g=\iota \circ f \circ \iota^{-1} \circ g \circ \iota^{-1}, \quad \forall f, g \in \operatorname{Diff}(M) ;
$$


(ii) the Hom-unit is $\iota \in \operatorname{Diff}(M)$;

(iii) the map $\operatorname{Ad}_{\iota}: \operatorname{Diff}(M) \rightarrow \operatorname{Diff}(M)$ is defined by $\operatorname{Ad}_{\iota}(f)=\iota \circ f \circ \iota^{-1}$, for all $f \in \operatorname{Diff}(M)$.

Let $\left(G, \diamond, e_{\Phi}, \Phi\right)$ be a Hom-Lie group and $T G$ be the tangent bundle of the manifold $G$. Let us denote by $\Phi^{!} T G$, the pullback bundle of the tangent bundle $T G$ along the diffeomorphism $\Phi: G \rightarrow G$. Then we have the following one-to-one correspondence.

Lemma 3.5. There is a one-to-one correspondence between the space of sections of the tangent bundle $T G$ (i.e., $\Gamma(T G))$ and the space of sections of the pullback bundle $\Phi^{!} T G\left(\right.$ i.e., $\left.\Gamma\left(\Phi^{!} T G\right)\right)$.

Proof. Let $X \in \Gamma(T G)$, then define a smooth map $x: G \rightarrow T G$ by $x=X \circ \Phi$. Let us consider the set $\Gamma_{\Phi !}(T G)=\{x: G \rightarrow T G \mid x=X \circ \Phi\}$. Since the map $\Phi: G \rightarrow G$ is a diffeomorphism, there is a one-to-one correspondence between the sets $\Gamma(T G)$ and $\Gamma_{\Phi !}(T G)$ :

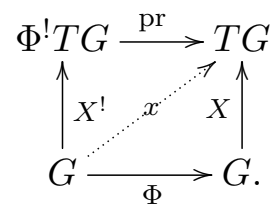

Note that there exists a unique $X^{!}$such that $x=\operatorname{pr} \circ X^{!}$. Hence, there is a one-to-one correspondence between $\Gamma(T G)$ with $\Gamma\left(\Phi^{!} T G\right)$.

For $X \in \Gamma(T G)$, we denote the corresponding pullback section by $X^{!} \in \Gamma\left(\Phi^{!} T G\right)$. Through this paper, we identify $X^{!} \in \Gamma\left(\Phi^{!} T G\right)$ by $x \in \Gamma_{\Phi^{!}}(T G)$. Let us observe that if we define

$$
x(f)=X(f) \circ \Phi, \quad \forall f \in C^{\infty}(G),
$$

then $\Gamma_{\Phi^{!}}(T G)$ can be identified with the set of $\left(\Phi^{*}, \Phi^{*}\right)$-derivations $\operatorname{Der}_{\Phi^{*}, \Phi^{*}}\left(C^{\infty}(G)\right)$ on $C^{\infty}(G)$, i.e., for all $f, g \in C^{\infty}(G), x \in \Gamma_{\Phi !}(T G)$, we have

$$
x(f g)=x(f) \Phi^{*}(g)+\Phi^{*}(f) x(g) .
$$

Thus, the space of sections $\Gamma\left(\Phi^{!} T G\right)$ can be identified with the space of $\left(\Phi^{*}, \Phi^{*}\right)$-derivations of on $C^{\infty}(G)$, i.e., $\operatorname{Der}_{\Phi^{*}, \Phi^{*}}\left(C^{\infty}(G)\right)$. In the following theorem, we define a Hom-Lie algebra structure on the space of sections $\Gamma\left(\Phi^{!} T G\right)$.

Theorem 3.6. Let $G$ be a smooth manifold. Then $\left(\Gamma(\Phi ! T G),[\cdot, \cdot]_{\Phi}, \phi\right)$ is a Hom-Lie algebra, where the Hom-Lie bracket $[\cdot, \cdot]_{\Phi}$ and the map $\phi: \Gamma\left(\Phi^{!} T G\right) \rightarrow \Gamma\left(\Phi^{!} T G\right)$ are defined as follows:

$$
\begin{aligned}
& \phi(x)=\left(\Phi^{-1}\right)^{*} \circ x \circ \Phi^{*}, \\
& {[x, y]_{\Phi}=\left(\Phi^{-1}\right)^{*} \circ x \circ\left(\Phi^{-1}\right)^{*} \circ y \circ \Phi^{*}-\left(\Phi^{-1}\right)^{*} \circ y \circ\left(\Phi^{-1}\right)^{*} \circ x \circ \Phi^{*},}
\end{aligned}
$$

for any $x, y \in \Gamma\left(\Phi^{!} T G\right)$.

Proof. Let $x, y \in \Gamma\left(\Phi^{!} T G\right)$ and $f, g \in C^{\infty}(G)$. Then by (3.3) and (3.4), we get

$$
\begin{aligned}
\phi(x)(f g) & =\left(\Phi^{-1}\right)^{*} \circ x \circ \Phi^{*}(f g) \\
& =\left(x(f \circ \Phi) \circ \Phi^{-1}\right)(g \circ \Phi)+(f \circ \Phi)\left(x(g \circ \Phi) \circ \Phi^{-1}\right) \\
& =\phi(x)(f)(g \circ \Phi)+(f \circ \Phi) \phi(x)(g) \\
& =\phi(x)(f) \Phi^{*}(g)+\Phi^{*}(f) \phi(x)(g),
\end{aligned}
$$


which implies that $\phi(x)$ is a $\left(\Phi^{*}, \Phi^{*}\right)$-derivation on $C^{\infty}(G)$ and hence, $\phi(x) \in \Gamma\left(\Phi^{!} T G\right)$. Next, for any $x, y \in \Gamma\left(\Phi^{!} T G\right)$ and $f, g \in C^{\infty}(G)$, we have

$$
\begin{aligned}
{[x, y]_{\Phi}(f g)=} & \left(\Phi^{-1}\right)^{*} \circ x \circ\left(\Phi^{-1}\right)^{*} \circ y \circ \Phi^{*}(f g)-\left(\Phi^{-1}\right)^{*} \circ y \circ\left(\Phi^{-1}\right)^{*} \circ x \circ \Phi^{*}(f g) \\
= & x\left(y((f \circ \Phi)(g \circ \Phi)) \circ \Phi^{-1}\right) \circ \Phi^{-1}-y\left(x((f \circ \Phi)(g \circ \Phi)) \circ \Phi^{-1}\right) \circ \Phi^{-1} \\
= & x\left(\left(y(f \circ \Phi)\left(g \circ \Phi^{2}\right)+\left(f \circ \Phi^{2}\right) y(g \circ \Phi)\right) \circ \Phi^{-1}\right) \circ \Phi^{-1} \\
& -y\left(\left(x(f \circ \Phi)\left(g \circ \Phi^{2}\right)+\left(f \circ \Phi^{2}\right) x\left(g \circ \Phi^{-1}\right)\right) \circ \Phi^{-1}\right) \circ \Phi^{-1} \\
= & \left(x\left(y(f \circ \Phi) \circ \Phi^{-1}\right) \circ \Phi^{-1}\right)(g \circ \Phi)-\left(y\left(x(f \circ \Phi) \circ \Phi^{-1}\right) \circ \Phi^{-1}\right)(g \circ \Phi) \\
& +(f \circ \Phi)\left(x\left(y(g \circ \Phi) \circ \Phi^{-1}\right) \circ \Phi^{-1}\right)-(f \circ \Phi)\left(y\left(x(g \circ \Phi) \circ \Phi^{-1}\right) \circ \Phi^{-1}\right),
\end{aligned}
$$

and

$$
\begin{aligned}
{[x, y]_{\Phi}(f) \Phi^{*}(g)+\Phi^{*}(f)[x, y]_{\Phi}(g) } & \\
= & \left(x\left(y(f \circ \Phi) \circ \Phi^{-1}\right) \circ \Phi^{-1}\right)(g \circ \Phi)-\left(y\left(x(f \circ \Phi) \circ \Phi^{-1}\right) \circ \Phi\right)(g \circ \Phi) \\
& +(f \Phi)\left(x\left(y\left(g \Phi^{-1}\right) \Phi^{-1}\right) \Phi\right)-(f \Phi)\left(y\left(x\left(g \Phi^{-1}\right) \Phi^{-1}\right) \Phi\right) .
\end{aligned}
$$

i.e.,

$$
[x, y]_{\Phi}(f g)=[x, y]_{\Phi}(f) \Phi^{*}(g)+\Phi^{*}(f)[x, y]_{\Phi}(g),
$$

which implies that $[x, y]_{\Phi} \in \Gamma\left(\Phi^{!} T G\right)$. Moreover, by (3.4) and (3.5), we get the following expressions:

$$
\phi[x, y]_{\Phi}=\left(\Phi^{-2}\right)^{*} \circ x \circ\left(\Phi^{-1}\right)^{*} \circ y \circ\left(\Phi^{2}\right)^{*}-\left(\Phi^{-2}\right)^{*} \circ y \circ\left(\Phi^{-1}\right)^{*} \circ x \circ\left(\Phi^{2}\right)^{*},
$$

and

$$
[\phi(x), \phi(y)]_{\Phi}=\left(\Phi^{-2}\right)^{*} \circ x \circ\left(\Phi^{-1}\right)^{*} \circ y \circ\left(\Phi^{2}\right)^{*}-\left(\Phi^{-2}\right)^{*} \circ y \circ\left(\Phi^{-1}\right)^{*} \circ x \circ\left(\Phi^{2}\right)^{*},
$$

which, in turn, implies that $\phi[x, y]_{\Phi}=[\phi(x), \phi(y)]_{\Phi}$.

Finally, we have

$$
\begin{aligned}
{\left[\phi(x),[y, z]_{\Phi}\right]_{\Phi}=} & \left(\Phi^{-2}\right)^{*} \circ x \circ\left(\Phi^{-1}\right)^{*} \circ y \circ\left(\Phi^{-1}\right)^{*} \circ z \circ\left(\Phi^{2}\right)^{*} \\
& -\left(\Phi^{-2}\right)^{*} \circ x \circ\left(\Phi^{-1}\right)^{*} \circ z \circ\left(\Phi^{-1}\right)^{*} \circ y \circ\left(\Phi^{2}\right)^{*} \\
& -\left(\Phi^{-2}\right)^{*} \circ y \circ\left(\Phi^{-1}\right)^{*} \circ z \circ\left(\Phi^{-1}\right)^{*} \circ x \circ\left(\Phi^{2}\right)^{*} \\
& +\left(\Phi^{-2}\right)^{*} \circ z \circ\left(\Phi^{-1}\right)^{*} \circ y \circ\left(\Phi^{-1}\right)^{*} \circ x \circ\left(\Phi^{2}\right)^{*} .
\end{aligned}
$$

Similarly, we have

$$
\begin{aligned}
{\left[\phi(y),[z, x]_{\Phi}\right]_{\Phi}=} & \left(\Phi^{-2}\right)^{*} \circ y \circ\left(\Phi^{-1}\right)^{*} \circ z \circ\left(\Phi^{-1}\right)^{*} \circ x \circ\left(\Phi^{2}\right)^{*} \\
& -\left(\Phi^{-2}\right)^{*} \circ y \circ\left(\Phi^{-1}\right)^{*} \circ x \circ\left(\Phi^{-1}\right)^{*} \circ z \circ\left(\Phi^{2}\right)^{*} \\
& -\left(\Phi^{-2}\right)^{*} \circ z \circ\left(\Phi^{-1}\right)^{*} \circ x \circ\left(\Phi^{-1}\right)^{*} \circ y \circ\left(\Phi^{2}\right)^{*} \\
& +\left(\Phi^{-2}\right)^{*} \circ x \circ\left(\Phi^{-1}\right)^{*} \circ z \circ\left(\Phi^{-1}\right)^{*} \circ y \circ\left(\Phi^{2}\right)^{*},
\end{aligned}
$$

and

$$
\begin{aligned}
{\left[\phi(z),[x, y]_{\Phi}\right]_{\Phi}=} & \left(\Phi^{-2}\right)^{*} \circ z \circ\left(\Phi^{-1}\right)^{*} \circ x \circ\left(\Phi^{-1}\right)^{*} \circ y \circ\left(\Phi^{2}\right)^{*} \\
& -\left(\Phi^{-2}\right)^{*} \circ z \circ\left(\Phi^{-1}\right)^{*} \circ y \circ\left(\Phi^{-1}\right)^{*} \circ x \circ\left(\Phi^{2}\right)^{*} \\
& -\left(\Phi^{-2}\right)^{*} \circ y \circ\left(\Phi^{-1}\right)^{*} \circ z \circ\left(\Phi^{-1}\right)^{*} \circ z \circ\left(\Phi^{2}\right)^{*} \\
& +\left(\Phi^{-2}\right)^{*} \circ y \circ\left(\Phi^{-1}\right)^{*} \circ x \circ\left(\Phi^{-1}\right)^{*} \circ z \circ\left(\Phi^{2}\right)^{*},
\end{aligned}
$$

which implies that

$$
\left[\phi(x),[y, z]_{\Phi}\right]_{\Phi}+\left[\phi(y),[z, x]_{\Phi}\right]_{\Phi}+\left[\phi(z),[x, y]_{\Phi}\right]_{\Phi}=0 .
$$

Therefore, $\left(\Gamma\left(\Phi^{!} T G\right),[\cdot, \cdot]_{\Phi}, \phi\right)$ is a Hom-Lie algebra. 


\subsection{The Hom-Lie algebra of a Hom-Lie group}

Let $\left(G, \diamond, e_{\Phi}, \Phi\right)$ be a Hom-Lie group. For $a \in G$, let us define a smooth map

$$
l_{a}: G \rightarrow G \quad \text { by } \quad l_{a}(b)=a \diamond b, \quad \forall b \in G \text {. }
$$

Then the smooth map $l_{a}: G \rightarrow G$ is a diffeomorphism (by Definition 3.1).

Definition 3.7. Let $\left(G, \diamond, e_{\Phi}, \Phi\right)$ be a Hom-Lie group. A smooth section $x \in \Gamma\left(\Phi^{!} T G\right)$ is left-invariant if $x$ satisfies the following equation:

$$
x(f)(a)=x\left(f \circ l_{a} \circ \Phi^{-1}\right)\left(e_{\Phi}\right), \quad \forall a \in G, f \in C^{\infty}(G) .
$$

Let us denote by $\Gamma_{L}\left(\Phi^{!} T G\right)$, the space of all left-invariant sections of the pullback bundle $\Phi ! T G$. Next, we show that the space $\Gamma_{L}(\Phi ! T G)$ carries a Hom-Lie algebra structure. In fact, we prove that $\left(\Gamma_{L}(\Phi ! T G),[\cdot, \cdot]_{\Phi}, \phi\right)$ is a Hom-Lie subalgebra of the Hom-Lie algebra $\left(\Gamma(\Phi ! T G),[\cdot, \cdot]_{\Phi}, \phi\right)$.

Lemma 3.8. Let $\left(G, \diamond, e_{\Phi}, \Phi\right)$ be a Hom-Lie group and $x \in \Gamma\left(\Phi^{!} T G\right)$ be a left-invariant section. Then we have

$$
x(f \circ \Phi) \circ \Phi^{-1} \circ l_{\Phi^{-1}(a)}=x\left(f \circ l_{a}\right), \quad \forall a \in G .
$$

Proof. First, let us note that by using the Hom-associativity condition of the product $\diamond$, we get the following equation:

$$
\Phi \circ l_{\left(\Phi^{-2}(a) \diamond \Phi^{-1}(b)\right)}=l_{a} \circ l_{b}, \quad \forall a, b \in G,
$$

which implies that

$$
f \circ \Phi \circ l_{\left(\Phi^{-2}(a) \diamond \Phi^{-1}(b)\right)} \circ \Phi^{-1}=f \circ l_{a} \circ l_{b} \circ \Phi^{-1} .
$$

By using the left-invariant property (3.6) of the section $x$, we have

$$
x(f \circ \Phi) \circ \Phi^{-1} \circ l_{\Phi^{-1}(a)}(b)=x\left(f \circ \Phi \circ l_{\left(\Phi^{-2}(a) \diamond \Phi^{-1}(b)\right)} \circ \Phi^{-1}\right)\left(e_{\Phi}\right),
$$

and

$$
x\left(f \circ l_{a}\right)(b)=x\left(f \circ l_{a} \circ l_{b} \circ \Phi^{-1}\right)\left(e_{\Phi}\right) .
$$

Thus, by (3.8)-(3.10), we deduce that the desired identity (3.7) holds.

Theorem 3.9. The space $\Gamma_{L}(\Phi ! T G)$ of left-invariant sections of the pullback bundle $\Phi ! T G$ is a Hom-Lie subalgebra of the Hom-Lie algebra $\left(\Gamma(\Phi ! T G),[\cdot, \cdot]_{\Phi}, \phi\right)$.

Proof. First, let us prove that $\phi(x) \in \Gamma_{L}\left(\Phi^{!} T G\right)$ for any $x \in \Gamma_{L}\left(\Phi^{!} T G\right)$. By (3.4) and (3.6), we have

$$
\begin{aligned}
\phi(x)(f)(a) & =\left(\Phi^{-1}\right)^{*} \circ x \circ \Phi^{*}(f)(a)=x(f \circ \Phi)\left(\Phi^{-1}(a)\right) \\
& =x\left(f \circ \Phi \circ l_{\Phi^{-1}(a)} \Phi^{-1}\right)(e)=x\left(f \circ l_{a}\right)(e)=\phi(x)\left(f \circ l_{a} \circ \Phi^{-1}\right)(e)
\end{aligned}
$$

for all $x, y \in \Gamma_{L}\left(\Phi^{!} T G\right)$, and $a \in G$. This, in turn, implies that $\phi(x) \in \Gamma_{L}\left(\Phi^{!} T G\right)$.

Now we prove that $[x, y]_{\Phi} \in \Gamma_{L}\left(\Phi^{!} T G\right)$. By (3.5) and (3.6), we have the following expressions:

$$
\begin{aligned}
{[x, y]_{\Phi}(f)(a) } & =x\left(y(f \circ \Phi) \circ \Phi^{-1}\right)\left(\Phi^{-1}(a)\right)-y\left(x(f \circ \Phi) \circ \Phi^{-1}\right)\left(\Phi^{-1}(a)\right) \\
& =x\left(y(f \circ \Phi) \circ \Phi^{-1} \circ l_{\Phi^{-1}(a)} \circ \Phi^{-1}\right)\left(e_{\Phi}\right)
\end{aligned}
$$




$$
-y\left(x(f \circ \Phi) \circ \Phi^{-1} \circ l_{\Phi^{-1}(a)} \circ \Phi^{-1}\right)\left(e_{\Phi}\right),
$$

and

$$
[x, y]_{\Phi}\left(f \circ l_{a} \circ \Phi^{-1}\right)\left(e_{\Phi}\right)=x\left(y\left(f \circ l_{a}\right) \circ \Phi^{-1}\right)\left(e_{\Phi}\right)-y\left(x\left(f \circ l_{a}\right) \circ \Phi^{-1}\right)\left(e_{\Phi}\right)
$$

for all $x, y \in \Gamma_{L}\left(\Phi^{!} T G\right)$ and $a \in G$. Thus, from Lemma 3.8, we have

$$
[x, y]_{\Phi}(f)(a)=[x, y]_{\Phi}\left(f \circ l_{a} \circ \Phi^{-1}\right)\left(e_{\Phi}\right),
$$

which implies that $[x, y]_{\Phi} \in \Gamma_{L}\left(\Phi^{!} T G\right)$. The proof is finished.

Remark 3.10. Let $\left(G, \diamond, e_{\Phi}, \Phi\right)$ be a Hom-Lie group. Then we get a Lie group structure $\left(G, \cdot, e_{\Phi}\right)$ equipped with the product $\cdot: G \times G \rightarrow G$ defined by $a \cdot b=\Phi^{-1}(a \diamond b)$ for all $a, b \in G$.

Lemma 3.11. Let $\left(G, \diamond, e_{\Phi}, \Phi\right)$ be a Hom-Lie group. Let $x$ be a section of $\Phi^{!} T G$ and $X$ be the corresponding section of $T G$. Then $x$ is left-invariant if and only if $X$ is a left-invariant vector field of the associated Lie group $\left(G, \cdot, e_{\Phi}\right)$ (by Remark 3.10).

Proof. If $x \in \Gamma_{L}\left(\Phi^{!} T G\right)$, then by the definition of a left-invariant section, we get

$$
x(f)(a)=x\left(f \circ l_{a} \circ \Phi^{-1}\right)\left(e_{\Phi}\right), \quad \forall f \in C^{\infty}(G), \quad a \in G .
$$

Let $X$ be the corresponding section of $T G$, i.e., $x=X \circ \Phi$. Then we obtain the following expression:

$$
X(f)(\Phi(a))=X\left(f \circ l_{a} \circ \Phi^{-1}\right)\left(e_{\Phi}\right)=X\left(f \circ L_{\Phi(a)}\right)\left(e_{\Phi}\right),
$$

where $L_{\Phi(a)}(b)=\Phi(a) \cdot b=a \diamond \Phi^{-1}(b)$. Thus, $X$ is a left invariant vector field of the Lie group $\left(G, \cdot, e_{\Phi}\right)$.

Similarly, if $X \in \Gamma(T G)$ is a left-invariant vector field of the Lie group $\left(G, \cdot, e_{\Phi}\right)$, then we can deduce that the corresponding section $x \in \Gamma\left(\Phi^{!} T G\right)$ is left-invariant. We omit the details.

Let $\left(G, \diamond, e_{\Phi}, \Phi\right)$ be a Hom-Lie group. Let us denote by $\mathfrak{g}^{!}$, the fibre of $e_{\Phi}$ in the pullback bundle $\Phi^{!} T G$. Notice that $\mathfrak{g}=T_{e_{\Phi}} G=\Phi^{!} T_{e_{\Phi}} G=\mathfrak{g}^{!}\left(\right.$since, $\left.\Phi\left(e_{\Phi}\right)=e_{\Phi}\right)$. Then by Lemma 3.11, $\mathfrak{g}^{!}$is in one-to-one correspondence with $\Gamma_{L}\left(\Phi^{!} T G\right)$. With this in mind, it is natural to define a bracket $[\cdot, \cdot]_{\mathfrak{g}}$ ! and a vector space isomorphism $\phi_{\mathfrak{g} !}: \mathfrak{g}^{!} \rightarrow \mathfrak{g}^{!}$as follows:

$$
\begin{aligned}
& {\left[x\left(e_{\Phi}\right), y\left(e_{\Phi}\right)\right]_{\mathfrak{g} !}=[x, y]_{\Phi}\left(e_{\Phi}\right),} \\
& \phi_{\mathfrak{g}^{!}}\left(x\left(e_{\Phi}\right)\right)=(\phi(x))\left(e_{\Phi}\right),
\end{aligned}
$$

for all $x, y \in \Gamma_{L}\left(\Phi^{!} T G\right)$. It follows that the triple $\left(\mathfrak{g}^{!},[\cdot, \cdot]_{\mathfrak{g}^{!}}, \phi_{\mathfrak{g}^{!}}\right)$is a Hom-Lie algebra and it is isomorphic to the Hom-Lie algebra $\left(\Gamma_{L}\left(\Phi^{!} T G\right),[\cdot, \cdot]_{\Phi}, \phi\right)$.

Lemma 3.12. Let $\left(G, \diamond, e_{\Phi}, \Phi\right)$ be a Hom-Lie group. If $x, y \in \Gamma_{L}\left(\Phi^{!} T G\right)$, and $X, Y$ are the corresponding left-invariant vector fields of the Lie group $\left(G, \cdot, e_{\Phi}\right)$, then we obtain the following identities:

$$
\begin{aligned}
& {[x, y]_{\Phi}\left(e_{\Phi}\right)=\Phi_{* e_{\Phi}}\left([X, Y]\left(e_{\Phi}\right)\right),} \\
& \phi(x)\left(e_{\Phi}\right)=\Phi_{* e_{\Phi}}\left(X\left(e_{\Phi}\right)\right) .
\end{aligned}
$$

Here, the map $\Phi_{*}: T G \rightarrow T G$ is the differential of the smooth map $\Phi: G \rightarrow G$. 
Proof. By (3.2) and (3.4), we get

$$
\begin{aligned}
{[x, y]_{\Phi}(f) } & =x\left(y(f \circ \Phi) \circ \Phi^{-1}\right) \circ \Phi^{-1}-y\left(x(f \circ \Phi) \circ \Phi^{-1}\right) \circ \Phi^{-1} \\
& =x(Y(f \circ \Phi)) \circ \Phi^{-1}-y(X(f \circ \Phi)) \circ \Phi^{-1} \\
& =X(Y(f \circ \Phi))-Y(X(f \circ \Phi))=[X, Y](f \circ \Phi)
\end{aligned}
$$

for all $f \in C^{\infty}(G)$. Let $Z \in \Gamma(T G)$ be the corresponding section of $[x, y]_{\Phi} \in \Gamma\left(\Phi^{!} T G\right)$, i.e., $Z \circ \Phi=[x, y]_{\Phi}$. Then, we get the following expressions:

$$
Z(f)=[x, y]_{\Phi}(f) \circ \Phi^{-1}=([X, Y](f \circ \Phi)) \circ \Phi^{-1},
$$

and

$$
Z(f)\left(e_{\Phi}\right)=[x, y]_{\Phi}(f)\left(e_{\Phi}\right)=[X, Y](f \circ \Phi)\left(e_{\Phi}\right)=\Phi_{* e_{\Phi}}\left([X, Y]\left(e_{\Phi}\right)\right)(f) .
$$

Thus, $Z\left(e_{\Phi}\right)=\Phi_{* e_{\Phi}}\left([X, Y]\left(e_{\Phi}\right)\right)$ and we deduce that $[x, y]_{\Phi}\left(e_{\Phi}\right)=\Phi_{* e_{\Phi}}\left([X, Y]\left(e_{\Phi}\right)\right)$.

Next, let us assume that $W \in \Gamma(T G)$ is the corresponding section of $\phi(x) \in \Gamma\left(\Phi^{!} T G\right)$. Since $\phi(x)\left(e_{\Phi}\right)=W\left(e_{\Phi}\right)$, we have

$$
W(f)\left(e_{\Phi}\right)=\phi(x)(f)\left(e_{\Phi}\right)=x(f \circ \Phi)\left(e_{\Phi}\right)=X(f \circ \Phi)\left(e_{\Phi}\right),
$$

which implies that $\phi(x)\left(e_{\Phi}\right)=W_{e_{\Phi}}=\Phi_{* e_{\Phi}}\left(X\left(e_{\Phi}\right)\right)$.

At the end of this subsection, we show that every regular Hom-Lie algebra is integrable.

Definition 3.13. A Hom-Lie group $\left(G, \diamond, e_{\Phi}, \Phi\right)$ is called simply connected Hom-Lie group if the underlying manifold $G$ is a simply connected topological space.

Theorem 3.14. Let $\left(\mathfrak{g},[\cdot, \cdot]_{\mathfrak{g}}, \phi_{\mathfrak{g}}\right)$ be a regular Hom-Lie algebra. Then there exists a unique simply connected Hom-Lie group $\left(G, \diamond, e_{\Phi}, \Phi\right)$ such that $\mathfrak{g}=\mathfrak{g}^{!}$and $\phi_{\mathfrak{g}}=\Phi_{* e_{\Phi}}=\phi_{\mathfrak{g} !}$, where $\left(\mathfrak{g}^{!},[\cdot, \cdot]_{\mathfrak{g}} !, \phi_{\mathfrak{g} !}\right)$ is the associated Hom-Lie algebra.

Proof. For the Lie algebra $\left(\mathfrak{g},[\cdot, \cdot]_{\text {Lie }}\right)$ given in Lemma 2.2 , it is easy to see that $\phi_{\mathfrak{g}}$ is a Lie algebra isomorphism of $\left(\mathfrak{g},[\cdot, \cdot]_{\text {Lie }}\right)$.

We have a unique simply connected Lie group $(G, \cdot)$ such that $\left(\mathfrak{g},[\cdot, \cdot]_{\text {Lie }}\right)$ is the Lie algebra of $(G, \cdot)$. Since $\phi_{\mathfrak{g}}$ is a Lie algebra isomorphism of $\left(\mathfrak{g},[\cdot, \cdot]_{\text {Lie }}\right)$ and $G$ is a simply connected Lie group, we have a unique isomorphism $\Phi$ of the Lie group $(G, \cdot)$ such that $\Phi_{* e}=\phi_{\mathfrak{g}}$. By Example 3.2, the tuple $\left(G, \diamond, e_{\Phi}, \Phi\right)$ is a Hom-Lie group. Finally, by Lemma 3.12, it follows that

$$
[x, y]_{\mathfrak{g} !}=\Phi_{* e}[x, y]_{\text {Lie }}=\phi_{\mathfrak{g}}[x, y]_{\text {Lie }}=[x, y]_{\mathfrak{g}}, \quad \phi_{\mathfrak{g} !}(x)=\Phi_{* e}(x)=\phi_{\mathfrak{g}}(x), \quad \forall x, y \in \mathfrak{g} !
$$

which implies that $\phi_{\mathfrak{g} !}=\Phi_{* e_{\Phi}}$.

\subsection{One-parameter Hom-Lie subgroups}

Let $\left(G, \diamond, e_{\Phi}, \Phi\right)$ be a Hom-Lie group and $\left(\mathfrak{g}^{!},[\cdot, \cdot]_{\mathfrak{g} !}, \phi_{\mathfrak{g} !}\right)$ be its Hom-Lie algebra. Then we define one-parameter Hom-Lie subgroups of $\left(G, \diamond, e_{\Phi}, \Phi\right)$ and prove that there is a one-to-one correspondence between elements of $\mathfrak{g}^{!}$and one-parameter Hom-Lie subgroups of $\left(G, \diamond, e_{\Phi}, \Phi\right)$.

Definition 3.15. A weak homomorphism of Hom-Lie groups

$$
\sigma^{!}:(R,+, 0, \mathrm{Id}) \rightarrow\left(G, \diamond, e_{\Phi}, \Phi\right)
$$

is called a one-parameter Hom-Lie subgroup of the Hom-Lie group $\left(G, \diamond, e_{\Phi}, \Phi\right)$. 
Theorem 3.16. Let $\left(G, \diamond, e_{\Phi}, \Phi\right)$ be a Hom-Lie group. Then $\sigma^{!}:(R,+, 0, \mathrm{Id}) \rightarrow\left(G, \diamond, e_{\Phi}, \Phi\right)$ is a one-parameter Hom-Lie subgroup of the Hom-Lie group $\left(G, \diamond, e_{\Phi}, \Phi\right)$ if and only if there exists a unique $x \in \mathfrak{g}^{!}$such that $\sigma^{!}(t)=\Phi(\exp (t x))$, where $\exp$ is the exponential map of the Lie group $\left(G, \cdot, e_{\Phi}\right)$.

Proof. For all $x \in \mathfrak{g}^{\text {!' we have }}$

$$
\Phi\left(\sigma^{!}(t+s)\right)=\Phi(\Phi(\exp ((t+s) x)))=\Phi(\exp (t x) \diamond \exp (s x))=\sigma^{!}(t) \diamond \sigma^{!}(s),
$$

which implies that $\sigma^{!}(t)=\Phi(\exp (t x))$ is a one-parameter Hom-Lie subgroup of the Hom-Lie group $\left(G, \diamond, e_{\Phi}, \Phi\right)$. For different $x \in \mathfrak{g}^{\text {! }}$, we get a different one-parameter Hom-Lie subgroup.

Now, let us assume that $\sigma^{!}:(R,+, 0, \mathrm{Id}) \rightarrow\left(G, \diamond, e_{\Phi}, \Phi\right)$ is a one-parameter Hom-Lie subgroup of the Hom-Lie group $\left(G, \diamond, e_{\Phi}, \Phi\right)$. Then, for all $t, s \in \mathbb{R}$,

$$
\Phi^{-1}\left(\sigma^{!}(t+s)\right)=\Phi^{-2}\left(\sigma^{!}(t) \diamond \sigma^{!}(s)\right)=\Phi^{-1}\left(\sigma^{!}(t) \cdot \sigma^{!}(s)\right)=\Phi^{-1}\left(\sigma^{!}(t)\right) \cdot \Phi^{-1}\left(\sigma^{!}(s)\right),
$$

which implies that $\Phi^{-1}\left(\sigma^{!}(t)\right)$ is a one-parameter Lie subgroup of the Lie group $\left(G, \cdot, e_{\Phi}\right)$ (defined in Remark 3.10). Thus there exists a unique $x \in \mathfrak{g}^{!}$, such that $\sigma^{!}(t)=\Phi(\exp (t x))$. The proof is finished.

By Theorem 3.16, one-parameter Hom-Lie subgroups of Hom-Lie group $\left(G, \diamond, e_{\Phi}, \Phi\right)$ are in one-to-one correspondence with $\mathfrak{g}$ !. We denote by $\sigma_{x}^{!}(t)$ the one-parameter Hom-Lie subgroup of the Hom-Lie group $\left(G, \diamond, e_{\Phi}, \Phi\right)$, which corresponds with $x$.

\subsection{The Hexp map}

Let $\left(G, \diamond, e_{\Phi}, \Phi\right)$ be a Hom-Lie group and $\mathfrak{g}$ ! be the fibre of the pullback bundle $\Phi ! T G$ at $e_{\Phi}$. Also, let us assume that $\sigma^{!}:(R,+, 0, \mathrm{Id}) \rightarrow\left(G, \diamond, e_{\Phi}, \Phi\right)$ is a one-parameter Hom-Lie subgroup of the Hom-Lie group $\left(G, \diamond, e_{\Phi}, \Phi\right)$.

Then, let us define a map Hexp: $\mathfrak{g}^{!} \rightarrow G$ by

$$
\operatorname{Hexp}(x)=\sigma_{x}^{!}(1), \quad \forall x \in \mathfrak{g} !
$$

Theorem 3.17. Let $\left(G, \diamond, e_{\Phi}, \Phi\right)$ be a Hom-Lie group and $\left(\mathfrak{g}^{!},[\cdot, \cdot]_{\mathfrak{g} !}, \Phi_{\mathfrak{g}^{!}}\right)$be the associated HomLie algebra. Then the Hom-Lie bracket $[\cdot, \cdot]_{\mathfrak{g} !}$ can be expressed in terms of the Hexp: $\mathfrak{g}^{!} \rightarrow G$ map as follows:

$$
[x, y]_{\mathfrak{g} !}=\left.\frac{\mathrm{d}}{\mathrm{d} t} \frac{\mathrm{d}}{\mathrm{d} s}\right|_{t=0, s=0}\left(\Phi^{-3}(\operatorname{Hexp}(s x) \diamond \operatorname{Hexp}(t y)) \diamond \Phi^{-2}(\operatorname{Hexp}(-s x))\right) \diamond \Phi^{-1}(\operatorname{Hexp}(-t y)),
$$

for any $x, y \in \mathfrak{g}$ !.

Proof. Let us denote

$$
\Omega_{(x, y)}(t, s):=\left(\Phi^{-3}(\operatorname{Hexp}(s x) \diamond \operatorname{Hexp}(t y)) \diamond \Phi^{-2}(\operatorname{Hexp}(-s x))\right) \diamond \Phi^{-1}(\operatorname{Hexp}(-t y))
$$

for all $x, y \in \mathfrak{g}^{!}$. From Remark 3.10 , the triple $\left(G, \cdot, e_{\Phi}\right)$ is a Lie group and $\mathfrak{g}=\mathfrak{g}$ !, where $\left(\mathfrak{g},[\cdot, \cdot]_{\mathfrak{g}}\right)$ is the Lie algebra of the Lie group $\left(G, \cdot, e_{\Phi}\right)$. Next, we use (3.11), Theorem 3.16, and Lemma 3.12 to obtain the following expression:

$$
\begin{aligned}
\left.\frac{\mathrm{d}}{\mathrm{d} t} \frac{\mathrm{d}}{\mathrm{d} s}\right|_{t=0, s=0} \Omega_{(x, y)}(t, s) & =\left.\frac{\mathrm{d}}{\mathrm{d} t} \frac{\mathrm{d}}{\mathrm{d} s}\right|_{t=0, s=0} \Phi(\exp (s x) \cdot \exp (t y) \cdot \exp (-s x) \cdot \exp (-t y)) \\
& =\Phi_{* e_{\Phi}}[x, y]_{\mathfrak{g}}=[x, y]_{\mathfrak{g} !} .
\end{aligned}
$$

The proof is finished. 
Example 3.18. Let $V$ be a real vector space. Let us recall from Example 3.3 that the tuple $\left(\mathrm{GL}(V), \diamond, \beta, \operatorname{Ad}_{\beta}\right)$ is a Hom-Lie group. Then the triple $\left(\mathfrak{g r}(V),[\cdot, \cdot]_{\mathfrak{g r}(V)}, \Psi_{\mathfrak{g r}(V)}\right)$ is the associated Hom-Lie algebra, where the bracket is given by

$$
[x, y]_{\mathfrak{g l}(V)}=\beta \circ x \circ \beta^{-1} \circ y \circ \beta^{-1}-\beta \circ y \circ \beta^{-1} \circ x \circ \beta^{-1},
$$

and $\Psi_{\mathfrak{g l}(V)}(x)=\beta \circ x \circ \beta^{-1}$.

Proposition 3.19. A map $f:\left(G, \diamond_{G}, e_{\Phi}, \Phi\right) \rightarrow\left(H, \diamond_{H}, e_{\Psi}, \Psi\right)$ is a weak homomorphism of Hom-Lie groups if and only if $f:\left(G, \cdot_{G}, e_{\Phi}\right) \rightarrow\left(H,{ }_{H}, e_{\Psi}\right)$ is a Lie group homomorphism.

Proof. Let us assume that $f:\left(G, \diamond_{G}, e_{\Phi}, \Phi\right) \rightarrow\left(H, \diamond_{H}, e_{\Psi}, \Psi\right)$ is a weak homomorphism of Hom-Lie groups. Then, we have

$$
f\left(\Phi^{-1}(a) \diamond_{G} \Phi^{-1}(b)\right)=\Psi^{-1}(f(a) \diamond f(b)), \quad \forall a, b \in G,
$$

which implies that $f:\left(G, \cdot_{G}, e_{\Phi}\right) \rightarrow\left(H,{ }_{H}, e_{\Psi}\right)$ is a Lie group homomorphism.

Conversely, let $f:\left(G, \cdot_{G}, e_{\Phi}\right) \rightarrow\left(H, \cdot H, e_{\Psi}\right)$ be a Lie group homomorphism. Then, by (3.12), we deduce that $f$ is a weak homomorphism from $\left(G, \diamond_{G}, e_{\Phi}, \Phi\right)$ to $\left(H, \diamond_{H}, e_{\Psi}, \Psi\right)$.

Theorem 3.20. Let $f:\left(G, \diamond_{G}, e_{\Phi}, \Phi\right) \rightarrow\left(H, \diamond_{H}, e_{\Psi}, \Psi\right)$ be a weak homomorphism of Hom-Lie groups. For any $x \in \mathfrak{g}^{\text {!, we have }}$

$$
f \circ \sigma_{x}^{!}=\sigma_{y}^{!},
$$

where $y=\Psi_{* e_{\Psi}}^{-1} f_{* e_{\Phi}} \Phi_{* e_{\Phi}}(x)$ and $\sigma_{x}^{!}, \sigma_{y}^{!}$are the one-parameter Hom-Lie subgroups of the HomLie groups $\left(G, \diamond_{G}, e_{\Phi}, \Phi\right)$ and $\left(H, \diamond_{H}, e_{\Psi}, \Psi\right)$ determined by $x$ and $y$ respectively.

Proof. By the definition of the one-parameter Hom-Lie subgroup $\sigma_{x}^{!}$, it follows that

$$
f\left(\sigma_{x}^{!}(t+s)\right)=f\left(\Phi^{-1}\left(\sigma_{x}^{!}(t)\right) \diamond_{G} \Phi^{-1}\left(\sigma_{x}^{!}(s)\right)\right)=\Psi^{-1}\left(f\left(\left(\sigma_{x}^{!}(t)\right)\right) \diamond_{H} f\left(\left(\sigma_{x}^{!}(s)\right)\right)\right),
$$

i.e.,

$$
\Psi \circ f \circ \sigma_{x}^{!}(t+s)=f \circ \sigma_{x}^{!}(t) \diamond_{H} f \circ \sigma_{x}^{!}(s) .
$$

Thus, $f \circ \sigma_{x}^{!}$is a one-parameter Hom-Lie subgroup of the Hom-Lie group $\left(H, \diamond_{H}, e_{\Psi}, \Psi\right)$.

From Theorem 3.16, we obtain the following expressions

$$
f \circ \sigma_{x}^{!}(t)=f(\Phi(\exp (t x)))
$$

and

$$
f(\Phi(\exp (t x)))=\exp \left(t f_{* e_{\Phi}} \Phi_{* e_{\Phi}}(x)\right)=\Psi\left(\exp \left(t \Psi_{* e_{\Psi}}^{-1} f_{* e_{\Phi}} \Phi_{* e_{\Phi}}(x)\right)\right),
$$

which implies that $f \circ \sigma_{x}^{!}(t)=\sigma_{y}^{!}(t)$, where $y=\Psi_{* e_{\Psi}}^{-1} f_{* e_{\Phi}} \Phi_{* e_{\Phi}}(x)$.

Let $f:\left(G, \diamond_{G}, e_{\Phi}, \Phi\right) \rightarrow\left(H, \diamond_{H}, e_{\Psi}, \Psi\right)$ be a weak homomorphism of Hom-Lie groups. Then, we define a map $f_{\triangleright}: \mathfrak{g}^{!} \rightarrow \mathfrak{h}^{!}$by

$$
f_{\triangleright}(x)=\Psi_{* e_{\Psi}}^{-1} f_{* e_{\Phi}} \Phi_{* e_{\Phi}}(x), \quad \forall x \in \mathfrak{g} !
$$

Theorem 3.21. With the above notations, the map $f_{\triangleright}:\left(\mathfrak{g}^{!},[\cdot, \cdot]_{\mathfrak{g}^{!}}, \phi_{\mathfrak{g}^{!}}\right) \rightarrow\left(\mathfrak{h} !,[\cdot, \cdot]_{\mathfrak{h} !}, \psi_{\mathfrak{h} !}\right)$ is a weak homomorphism of Hom-Lie algebras. 
Proof. For all $x, y \in \mathfrak{g}^{!}$, it follows that

$$
f_{\triangleright}\left([x, y]_{\mathfrak{g} !}\right)=\Psi_{* e_{\Psi}}^{-1} f_{* e_{\Phi}} \Phi_{* e_{\Phi}}\left([x, y]_{\mathfrak{g} !}\right)=\Psi_{* e_{\Psi}}^{-1} f_{* e_{\Phi}} \Phi_{* e_{\Phi}}^{2}([x, y]) .
$$

By using Proposition 3.19, we have

$$
\begin{aligned}
\Psi_{* e_{\Psi}}^{-1} f_{* e_{\Phi}} \Phi_{* e_{\Phi}}^{2}([x, y]) & =\left[\Psi_{* e_{\Psi}}^{-1} f_{* e_{\Phi}} \Phi_{* e_{\Phi}}^{2}(x), \Psi_{* e_{\Psi}}^{-1} f_{* e_{\Phi}} \Phi_{* e_{\Phi}}^{2}(y)\right] \\
& =\Psi_{* e_{\Psi}}^{2}\left[\Psi_{* e_{\Psi}}^{-1} f_{\triangleright} \Phi_{* e_{\Phi}}(x), \Psi_{* e_{\Psi}}^{-1} f_{\triangleright} \Phi_{* e_{\Phi}}(y)\right],
\end{aligned}
$$

i.e.,

$$
f_{\triangleright}\left([x, y]_{\Phi}\right)=\left[\Psi_{* e_{\Psi}}^{-1} f_{\triangleright} \Phi_{* e_{\Phi}}(x), \Psi_{* e_{\Phi}}^{-1} f_{\triangleright} \Phi_{* e_{\Phi}}(y)\right]_{\Psi} .
$$

Thus, $\psi f_{\triangleright}\left([x, y]_{\Phi}\right)=\left[f_{\triangleright} \phi(x), f_{\triangleright} \phi(y)\right]_{\Psi}$, which implies that $f_{\triangleright}:\left(\mathfrak{g}^{!},[\cdot, \cdot]_{\Phi}, \phi\right) \rightarrow\left(\mathfrak{h}^{!},[\cdot, \cdot]_{\Psi}, \psi\right)$ is a weak homomorphism of Hom-Lie algebras.

Theorem 3.22 (universality of the Hexp map). Let $f:\left(G, \diamond_{G}, e_{\Phi}, \Phi\right) \rightarrow\left(H, \diamond_{H}, e_{\Psi}, \Psi\right)$ be a weak homomorphism of Hom-Lie groups. Then,

$$
f(\operatorname{Hexp}(x))=\operatorname{Hexp}\left(f_{\triangleright}(x)\right), \quad \forall x \in \mathfrak{g}^{!},
$$

i.e., the following diagram commutes:

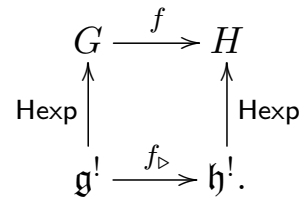

Proof. By the definition of Hexp, it follows that

$$
\operatorname{Hexp}\left(f_{\triangleright}(x)\right)=\Psi\left(\exp \left(\Psi_{* e_{\Psi}}^{-1} f_{* e_{\Phi}} \Phi_{* e_{\Phi}} x\right)\right),
$$

and

$$
f(\exp (x))=f(\Phi(\exp (x)))=\Psi\left(\exp \left(\Psi_{* e_{\Psi}}^{-1} f_{* e_{\Phi}} \Phi_{* e_{\Phi}} x\right)\right) .
$$

Thus we have $f(\operatorname{Hexp}(x))=\operatorname{Hexp}\left(f_{\triangleright}(x)\right)$, for all $x \in \mathfrak{g}$ !

\section{Actions of Hom-Lie groups and Hom-Lie algebras}

Let $\left(G, \diamond_{G}, e_{\Phi}, \Phi\right)$ be a Hom-Lie group and $M$ be a smooth manifold. Let $\theta: G \times M \rightarrow M$ be a smooth map that we denote by

$$
\theta(a, x)=a \odot x, \quad \forall a \in G, x \in M .
$$

Definition 4.1. The map $\theta: G \times M \rightarrow M$ is called an action of the Hom-Lie group $\left(G, \diamond_{G}, e_{\Phi}, \Phi\right)$ on the smooth manifold $M$ with respect to a map $\iota \in \operatorname{Diff}(M)$ if the following conditions are satisfied:

(i) $e \odot x=\iota(x), \forall x \in M$;

(ii) $\left(a \diamond_{G} b\right) \odot x=\Phi(a) \odot\left(\iota^{-1}(\Phi(b) \odot x)\right), \forall a, b \in G, x \in M$.

We denote this action by $(G, \theta, M, \iota)$. 
For all $a \in G$, define $L_{a}: M \rightarrow M$ by

$$
L_{a}(x)=a \odot x=\theta(a, x), \quad \forall x \in M .
$$

Since $L_{e_{\Phi}}=\iota \in \operatorname{Diff}(M)$ and $L_{\Phi^{-1}\left(a \diamond a^{-1}\right)}=L_{a} \circ \iota^{-1} \circ L_{a^{-1}}=\iota$, we have $L_{a} \circ \iota^{-1} \in \operatorname{Diff}(M)$, and thus $L_{a} \in \operatorname{Diff}(M)$. Let us define a map $L: G \rightarrow \operatorname{Diff}(M)$ by $L(a)=L_{a}$ for all $a \in G$.

Theorem 4.2. With the above notations, the map $\theta: G \times M \rightarrow M$ is an action of the HomLie group $\left(G, \diamond_{G}, e_{\Phi}, \Phi\right)$ on $M$ with respect to $\iota \in \operatorname{Diff}(M)$ if and only if the map $L: G \rightarrow$ $\operatorname{Diff}(M)$ is a weak homomorphism from the Hom-Lie group $\left(G, \diamond_{G}, e_{\Phi}, \Phi\right)$ to the Hom-Lie group $\left(\operatorname{Diff}(M), \diamond, \iota, \operatorname{Ad}_{\iota}\right)$.

Proof. Let us first assume that the map $\theta: G \times M \rightarrow \operatorname{Diff}(M)$ is an action of the Hom-Lie group $\left(G, \diamond_{G}, e_{\Phi}, \Phi\right)$ on $M$ with respect to the map $\iota \in \operatorname{Diff}(M)$. Then we have

$$
L\left(e_{\Phi}\right)=L_{e_{\Phi}}=\iota, \quad L(a \diamond b)(x)=\theta(a \diamond b, x)=L(\Phi(a)) \circ \iota^{-1} \circ L(\Phi(b))(x) .
$$

Thus, we have

$$
\operatorname{Ad}_{\iota} \circ L(a \diamond b)=L(\Phi(a)) \diamond L(\Phi(b)),
$$

which implies that the map $L:\left(G, \diamond_{G}, e_{\Phi}, \Phi\right) \rightarrow\left(\operatorname{Diff}(M), \diamond, \iota, \operatorname{Ad}_{\iota}\right)$ is a weak homomorphism of Hom-Lie groups.

Conversely, let us assume that $L:\left(G, \diamond_{G}, e_{\Phi}, \Phi\right) \rightarrow\left(\operatorname{Diff}(M), \diamond, \iota, \operatorname{Ad}_{\iota}\right)$ is a weak homomorphism of Hom-Lie groups. Then, it follows that

$$
\theta\left(e_{\Phi}, x\right)=L\left(e_{\Phi}\right)(x)=\iota(x), \quad \forall x \in M,
$$

and

$$
\left(\operatorname{Ad}_{\iota} \circ L(a \diamond b)\right)(x)=\iota \circ L(\Phi(a)) \circ \iota^{-1} \circ L(\Phi(b)) \circ \iota^{-1}(x)=\left(\iota \circ L(a \diamond b) \circ \iota^{-1}\right)(x),
$$

which implies that

$$
L(a \diamond b)(x)=L(\Phi(a)) \circ \iota^{-1} \circ L(\Phi(b))(x) .
$$

Therefore, we get the following identity:

$$
\theta(a \diamond b, x)=\Phi(a) \odot\left(\iota^{-1}(\Phi(b) \odot x)\right) .
$$

By (4.1) and (4.2), we deduce that $\theta: G \times M \rightarrow M$ is an action of the Hom-Lie group $\left(G, \diamond_{G}, e_{\Phi}, \Phi\right)$ on the smooth manifold $M$ with respect to $\iota \in \operatorname{Diff}(M)$.

If $\left(G, \diamond_{G}, e_{\Phi}, \Phi\right)$ is a Hom-Lie group, then let us define a map $\widetilde{\mathrm{Ad}}: G \times G \rightarrow G$ by

$$
\widetilde{\operatorname{Ad}}(a, b)=\Phi^{-1}\left(a \diamond_{G} b\right) \diamond_{G} a^{-1}, \quad \forall a, b \in G .
$$

Lemma 4.3. The map $\widetilde{\mathrm{Ad}}: G \times G \rightarrow G$ gives an action of the Hom-Lie group $\left(G, \diamond_{G}, e_{\Phi}, \Phi\right)$ on $G$ with respect to the map $\Phi \in \operatorname{Diff}(G)$.

Proof. For all $x \in G$, we have

$$
\widetilde{\operatorname{Ad}}\left(e_{\Phi}, x\right)=\Phi^{-1}\left(e_{\Phi} \diamond_{G} x\right) \diamond_{G} e_{\Phi}^{-1}=\Phi(x),
$$

and

$$
\widetilde{\operatorname{Ad}}\left(a \diamond_{G} b, x\right)=\Phi^{-1}\left(\left(a \diamond_{G} b\right) \diamond_{G} x\right) \diamond_{G}\left(a \diamond_{G} b\right)^{-1}, \quad \forall a, b \in G .
$$


Let us denote $a \odot b:=\widetilde{\operatorname{Ad}}(a, b)$, then we get the following expression:

$$
\begin{aligned}
\Phi(a) \odot\left(\Phi^{-1}(\Phi(b) \odot x)\right) & =\Phi(a) \odot\left(\Phi^{-1}\left(\Phi^{-1}\left(\Phi(b) \diamond_{G} x\right) \diamond_{G}(\Phi(b))^{-1}\right)\right) \\
& =\Phi(a) \odot\left(\left(\Phi^{-1}(b) \diamond_{G} \Phi^{-2}(x)\right) \diamond_{G} b^{-1}\right) \\
& =\Phi^{-1}\left(\Phi(a) \diamond_{G}\left(\left(\Phi^{-1}(b) \diamond_{G} \Phi^{-2}(x)\right) \diamond_{G} b^{-1}\right) \diamond_{G}(\Phi(a))^{-1}\right. \\
& =\left(a \diamond_{G}\left(\Phi^{-1}(b) \diamond_{G}\left(\Phi^{-3}(x) \diamond_{G}\left(\Phi^{-2}(b)\right)^{-1}\right)\right)\right) \diamond_{G}(\Phi(a))^{-1} \\
& =\left(\Phi^{-1}\left(a \diamond_{G} b\right) \diamond_{G}\left(\Phi^{-2}(x) \diamond_{G}\left(\Phi^{-1}(b)\right)^{-1}\right)\right) \diamond_{G} \Phi(a)^{-1} \\
& =\left(\left(\Phi^{-2}\left(a \diamond_{G} b\right) \diamond_{G} \Phi^{-2}(x)\right) \diamond_{G} b^{-1}\right) \diamond_{G} \Phi(a)^{-1} \\
& =\Phi^{-1}\left(\left(a \diamond_{G} b\right) \diamond x \diamond_{G}(a \diamond b)^{-1},\right.
\end{aligned}
$$

which implies that

$$
\begin{aligned}
\widetilde{\operatorname{Ad}}\left(a \diamond_{G} b, x\right) & =\Phi(a) \odot\left(\Phi^{-1}(\Phi(b) \odot x)\right) \\
& =\widetilde{\operatorname{Ad}}\left(\Phi(a),\left(\Phi^{-1}(\widetilde{\operatorname{Ad}}(\Phi(b), x))\right)\right), \quad \forall a, b, x \in G .
\end{aligned}
$$

Thus, the map $\widetilde{\mathrm{Ad}}: G \times G \rightarrow G$ gives an action of the Hom-Lie group $\left(G, \diamond_{G}, e_{\Phi}, \Phi\right)$ on the underlying manifold $G$ with respect to the map $\Phi \in \operatorname{Diff}(G)$.

Definition 4.4. Let $\left(G, \diamond_{G}, e_{\Phi}, \Phi\right)$ be a Hom-Lie group, $V$ be a vector space, and $\beta \in \mathrm{GL}(V)$. Then, a weak homomorphism of Hom-Lie groups

$$
\rho:\left(G, \diamond_{G}, e_{\Phi}, \Phi\right) \rightarrow\left(\mathrm{GL}(V), \diamond, \beta, \operatorname{Ad}_{\beta}\right)
$$

is called a representation of the Hom-Lie group $\left(G, \diamond_{G}, e_{\Phi}, \Phi\right)$ on the vector space $V$ with respect to $\beta \in \mathrm{GL}(V)$.

Let $\left(\mathfrak{g}^{!},[\cdot, \cdot] !, \phi_{\mathfrak{g} !}\right)$ be the Hom-Lie algebra of a Hom-Lie group $\left(G, \diamond_{G}, e_{\Phi}, \Phi\right)$. From Lemma 4.3, $\widetilde{\text { Ad }}$ gives an action of the Hom-Lie group $\left(G, \diamond_{G}, e_{\Phi}, \Phi\right)$ on $G$ with respect to the map $\Phi$. Now, let us denote $\widetilde{\operatorname{Ad}} a:=\widetilde{\operatorname{Ad}}(a, \cdot)$ for any $a \in G$. Then we observe that for all $a \in G$, the map $\widetilde{\mathrm{Ad}}_{a}: G \rightarrow G$ is a weak isomorphism of Hom-Lie groups. Let us denote by $\left(\widetilde{\operatorname{Ad}}_{a}\right)_{\triangleright}: \mathfrak{g} ! \rightarrow \mathfrak{g}$, the weak isomorphism of Hom-Lie algebra $\left(\mathfrak{g}^{!},[\cdot, \cdot]_{!}, \phi_{\mathfrak{g} !}\right)$, obtained by Theorem 3.21. Subsequently, we have the following lemma.

Lemma 4.5. The map $\widehat{\mathrm{Ad}}: G \rightarrow \mathrm{GL}\left(\mathfrak{g}^{!}\right)$, defined by

$$
\widehat{\operatorname{Ad}}(a)=\left(\widetilde{\operatorname{Ad}}_{a}\right)_{\triangleright}, \quad \forall a \in G
$$

is a weak homomorphism from $\left(G, \diamond_{G}, e_{\Phi}, \Phi\right)$ to $\left(\mathrm{GL}\left(\mathfrak{g}^{!}\right), \diamond, \phi_{\mathfrak{g}^{!}}, \operatorname{Ad}_{\phi_{\mathfrak{g}^{!}}}\right)$.

Proof. For all $a, b \in G$ and $x \in \mathfrak{g}^{!}$, it follows that

$$
\begin{aligned}
\widehat{\operatorname{Ad}}\left(a \diamond_{G} b\right)(x) & =\left(\widetilde{\operatorname{Ad}}_{a \diamond_{G} b}\right)_{\triangleright}(x) \\
& =\left.\frac{\mathrm{d}}{\mathrm{d} t}\right|_{t=0} \Phi^{-1}\left(\Phi^{-1}\left(\left(a \diamond_{G} b\right) \diamond_{G} \Phi(\exp (t x))\right) \diamond_{G}\left(a \diamond_{G} b\right)^{-1}\right)
\end{aligned}
$$

and

$$
\begin{aligned}
\widehat{\operatorname{Ad}}( & (a)) \circ \Phi_{*}^{-1} \circ \widehat{\operatorname{Ad}}(\Phi(b))(x) \\
& =\left.\widehat{\operatorname{Ad}}(\Phi(a)) \Phi_{*}^{-1} \frac{\mathrm{d}}{\mathrm{d} t}\right|_{t=0} \Phi^{-1}\left(\left(\Phi^{-1}\left(\Phi(b) \diamond_{G} \Phi(\exp (t x))\right) \diamond_{G}(\Phi(b))^{-1}\right)\right)
\end{aligned}
$$




$$
\begin{aligned}
& =\left.\frac{\mathrm{d}}{\mathrm{d} t}\right|_{t=0} \Phi^{-1}\left(\left(\Phi^{-1}\left(\Phi(a) \diamond_{G}\left(b \diamond_{G}\left(\Phi^{-1}\left(\exp (t x) \diamond_{G} b^{-1}\right)\right)\right) \diamond_{G}(\Phi(a))^{-1}\right)\right)\right) \\
& =\left.\frac{\mathrm{d}}{\mathrm{d} t}\right|_{t=0}\left(\Phi^{-1}(a) \diamond_{G}\left(\Phi^{-2}(b) \diamond_{G}\left(\Phi^{-3}\left(\exp (t x) \diamond_{G} b^{-1}\right)\right)\right) \diamond_{G} a^{-1}\right) \\
& =\left.\frac{\mathrm{d}}{\mathrm{d} t}\right|_{t=0}\left(\Phi^{-2}\left(a \diamond_{G} b\right) \diamond_{G}\left(\Phi^{-2}\left(\exp (t x) \diamond_{G} b^{-1}\right)\right)\right) \diamond_{G} a^{-1} \\
& =\left.\frac{\mathrm{d}}{\mathrm{d} t}\right|_{t=0}\left(\Phi^{-2}\left(a \diamond_{G} b\right) \diamond_{G} \Phi^{-1}(\exp (t x))\right) \diamond_{G} \Phi^{-1}\left(a \diamond_{G} b\right)^{-1},
\end{aligned}
$$

i.e.,

$$
\widehat{\operatorname{Ad}}\left(a \diamond_{G} b\right)=\widehat{\operatorname{Ad}}(\Phi(a)) \circ \Phi_{*}^{-1} \circ \widehat{\operatorname{Ad}}(\Phi(b)) .
$$

Thus, we have

$$
\Phi_{*} \circ \widehat{\operatorname{Ad}}\left(a \diamond_{G} b\right) \circ \Phi_{*}^{-1}=\Phi_{*} \circ \widehat{\operatorname{Ad}}(\Phi(a)) \circ \Phi_{*}^{-1} \circ \widehat{\operatorname{Ad}}(\Phi(b)) \circ \Phi_{*}^{-1},
$$

which implies that $\widehat{A d}$ is a Hom-Lie group (weak) homomorphism from $G$ to $\operatorname{GL}(\mathfrak{g}$ !).

From Lemma 4.5, every Hom-Lie group $\left(G, \diamond_{G}, e_{\Phi}, \Phi\right)$ has a representation on its Hom-Lie algebra $\left(\mathfrak{g}^{!},[\cdot, \cdot]_{\mathfrak{g} !}, \phi_{\mathfrak{g} !}\right)$ with respect to the map $\phi_{\mathfrak{g} !}$, which we call the "adjoint representation".

Let $(G, \theta, M, \iota)$ be an action of the Hom-Lie group $\left(G, \diamond_{G}, e_{\Phi}, \Phi\right)$. By Theorem 3.16, for any $x \in \mathfrak{g}^{\text {! }}$, we have a unique map $\sigma_{x}^{!}: \mathbb{R} \rightarrow G$. Now, we consider the curve $\gamma: \mathbb{R} \rightarrow M$ given by

$$
\gamma(t)=\Phi(\exp (t x)) \odot m, \quad \forall t \in \mathbb{R}, x \in \mathfrak{g}^{!}, m \in M .
$$

Then we get a section of the pullback bundle $\iota^{*} T M$ as follows:

$$
\widetilde{x}(m)=\left.\frac{\mathrm{d}}{\mathrm{d} t}\right|_{t=0} \gamma(t), \quad \forall m \in M .
$$

Thus, we have a map $\kappa: \mathfrak{g}^{!} \rightarrow \Gamma\left(\iota^{*} T M\right)$ given by $\kappa(x)=\widetilde{x}$ for all $x \in \mathfrak{g}^{!}$. We denote $\kappa(x)(m)$ simply by $x \bullet m$ for any $x \in \mathfrak{g}^{!}, m \in M$.

Definition 4.6. The map $\kappa: \mathfrak{g}^{!} \rightarrow \Gamma\left(\iota^{*} T M\right)$ is called the infinitesimal action of the Hom-Lie algebra $\mathfrak{g}^{!}$on the smooth manifold $M$ with respect to the map $\iota \in \operatorname{Diff}(M)$.

By Lemma 4.5 and Theorem 3.21, the map

$$
(\widehat{\mathrm{Ad}})_{\triangleright}:\left(\mathfrak{g}^{!},[\cdot, \cdot]_{\mathfrak{g} !}, \phi_{\mathfrak{g} !}\right) \rightarrow\left(\mathfrak{g l}\left(\mathfrak{g}^{!}\right),[\cdot, \cdot]_{\phi_{\mathfrak{g}} !}, \operatorname{Ad}_{\phi_{\mathfrak{g}} !}\right)
$$

is a weak homomorphism of Hom-Lie algebras. To simplify the notations, we denote $\widehat{a d}:=(\widehat{\mathrm{Ad}})_{\triangleright}$, then we have the following result.

Theorem 4.7. With the above notations, we have

$$
(\widehat{\operatorname{ad}}(x)) \bullet y=[x, y]_{\mathfrak{g}^{!}}, \quad \forall x, y \in \mathfrak{g}^{!} .
$$

Proof. By the definition of the infinitesimal action of Hom-Lie algebra $\mathfrak{g}^{\text {! }}$, we have

$$
\begin{aligned}
(\widehat{\operatorname{ad}}(x)) \bullet y & =\left.\frac{\mathrm{d}}{\mathrm{d} t}\right|_{t=0}(\Phi(\exp (t x)) \odot y) \\
& =\left.\left.\frac{d}{d t}\right|_{t=0} \frac{\mathrm{d}}{\mathrm{d} s}\right|_{s=0} \Phi^{-1}\left(\Phi^{-1}\left(\Phi(\exp (t x)) \diamond_{G} \Phi(\exp (s y))\right) \diamond_{G}(\Phi(\exp (t x)))^{-1}\right) \\
& =\left.\left.\frac{\mathrm{d}}{\mathrm{d} t}\right|_{t=0} \frac{\mathrm{d}}{\mathrm{d} s}\right|_{s=0} \Phi(\exp (t x)) \cdot \Phi(\exp (s y)) \cdot(\Phi(\exp (t x)))^{-1}=\Phi_{* e_{\Phi}}[x, y],
\end{aligned}
$$

which implies that $(\widehat{\operatorname{ad}}(x)) \bullet y=[x, y]_{\mathfrak{g} !}$. 
Proposition 4.8. Let $\left(G, \diamond_{G}, e_{\Phi}, \Phi\right)$ be a Hom-Lie group and $\mathfrak{g}$ ! be the Hom-Lie algebra of $G$. Then, we have

$$
\widehat{\operatorname{Ad}}(\operatorname{Hexp}(x))=\operatorname{Hexp}(\widehat{\operatorname{ad}}(x)), \quad \forall x \in \mathfrak{g}^{!} .
$$

Proof. The proof of the proposition follows from Theorem 3.22 and Lemma 4.5.

\section{Integration of the Hom-Lie algebra $\left(\mathfrak{g l}(V),[\cdot, \cdot]_{\beta}, \operatorname{Ad}_{\beta}\right)$}

Let $V$ be a vector space and $\beta \in \mathrm{GL}(V)$. Let us define a map $\mathfrak{e}_{\beta}: \mathfrak{g l}(V) \rightarrow \operatorname{GL}(V)$ by

$$
\mathfrak{e}_{\beta}^{A}=\beta+\beta \circ A \circ \beta^{-1}+\frac{1}{2 !} \beta \circ A \circ \beta^{-1} \circ A \circ \beta^{-1}+\cdots, \quad \forall A \in \mathfrak{g l}(V) .
$$

Let us denote by $e: \mathfrak{g l}(V) \rightarrow \mathrm{GL}(V)$, the usual exponential map. Then the map $\mathfrak{e}_{\beta}: \mathfrak{g l}(V) \rightarrow$ $\mathrm{GL}(V)$ can be written as follows:

$$
\mathfrak{e}_{\beta}^{A}=\beta \circ e^{A \circ \beta^{-1}}, \quad \forall A \in \mathfrak{g l}(V) .
$$

The map $\mathfrak{e}_{\beta}: \mathfrak{g l}(V) \rightarrow \mathrm{GL}(V)$ gives rise to a one-parameter Hom-Lie subgroup of the Hom-Lie group $\left(\mathrm{GL}(V), \diamond, \operatorname{Ad}_{\beta}\right)$. More precisely, for any $A \in \mathfrak{g l}(V)$, let us define a map $\sigma_{A}: \mathbb{R} \rightarrow \mathrm{GL}(V)$ by

$$
\sigma_{A}(t)=\mathfrak{e}_{\beta}^{t A}, \quad \forall t \in \mathbb{R} .
$$

Then we have the following lemma.

Lemma 5.1. With the above notations, $\sigma_{A}:(\mathbb{R},+, 0, \mathrm{Id}) \rightarrow\left(\mathrm{GL}(V), \diamond, \beta, \operatorname{Ad}_{\beta}\right)$ is a weak homomorphism of Hom-Lie groups, i.e.,

$$
\sigma_{A}(t+s)=\left(\operatorname{Ad}_{\beta^{-1}} \sigma_{A}(t)\right) \diamond\left(\operatorname{Ad}_{\beta^{-1}} \sigma_{A}(s)\right), \quad \forall t, s \in \mathbb{R} .
$$

Proof. By (5.1), we have

$$
\begin{aligned}
\sigma_{A}(t+s) & =\mathfrak{e}_{\beta}^{(t+s) A}=\beta \circ e^{(t+s)\left(A \beta^{-1}\right)}=\beta \circ\left(e^{t A \circ \beta^{-1}} e^{s A \circ \beta^{-1}}\right)=\mathfrak{e}_{\beta}^{t A} \circ \beta^{-1} \circ \mathfrak{e}_{\beta}^{s A} \\
& =\operatorname{Ad}_{\beta^{-1}} \mathfrak{e}_{\beta}^{s A} \diamond \operatorname{Ad}_{\beta^{-1}} \mathfrak{e}_{\beta}^{t A}=\left(\operatorname{Ad}_{\beta^{-1}} \sigma_{A}(t)\right) \diamond\left(\operatorname{Ad}_{\beta^{-1}} \sigma_{A}(s)\right),
\end{aligned}
$$

for all $t, s \in \mathbb{R}$.

Thus, for any $A \in \mathfrak{g l}(V)$, the map $\sigma_{A}:(\mathbb{R},+, 0, \mathrm{Id}) \rightarrow\left(\mathrm{GL}(V), \diamond, \beta, \operatorname{Ad}_{\beta}\right)$ is a one-parameter Hom-Lie subgroup of the Hom-Lie group $\left(\mathrm{GL}(V), \diamond, \beta, \mathrm{Ad}_{\beta}\right)$.

Proposition 5.2. Let us consider the Hom-Lie algebra $\left(\mathfrak{g l}(V),[\cdot, \cdot]_{\beta}, \operatorname{Ad}_{\beta}\right)$. Then the Hom-Lie bracket $[\cdot, \cdot]_{\beta}$ can be expressed as follows:

$$
[A, B]_{\beta}=\left.\frac{\mathrm{d}}{\mathrm{d} t} \frac{\mathrm{d}}{\mathrm{d} s}\right|_{t=0, s=0}\left(\left(\operatorname{Ad}_{\beta^{-3}}\left(\mathfrak{e}_{\beta}^{s A} \diamond \mathfrak{e}_{\beta}^{t B}\right)\right) \diamond \operatorname{Ad}_{\beta^{-2}}\left(\mathfrak{e}_{\beta}^{-s A}\right)\right) \diamond \operatorname{Ad}_{\beta^{-1}}\left(\mathfrak{e}_{\beta}^{-t B}\right) .
$$

Proof. By (5.1), it follows that

$$
\begin{aligned}
& \left.\frac{\mathrm{d}}{\mathrm{d} t} \frac{\mathrm{d}}{\mathrm{d} s}\right|_{t=0, s=0}\left(\left(\operatorname{Ad}_{\beta^{-3}}\left(\mathfrak{e}_{\beta}^{s A} \diamond \mathfrak{e}_{\beta}^{t B}\right)\right) \diamond \operatorname{Ad}_{\beta^{-2}}\left(\mathfrak{e}_{\beta}^{-s A}\right)\right) \diamond \operatorname{Ad}_{\beta^{-1}}\left(\mathfrak{e}_{\beta}^{-t B}\right) \\
& \quad=\left.\frac{\mathrm{d}}{\mathrm{d} t} \frac{\mathrm{d}}{\mathrm{d} s}\right|_{t=0, s=0}\left(\beta^{-2}\left(\beta^{3} e^{s A \beta^{-1}} e^{t B \beta^{-1}} e^{-s A \beta^{-1}} e^{-t B \beta^{-1}} \beta^{-2}\right) \beta^{2}\right) \\
& \quad=\left.\frac{\mathrm{d}}{\mathrm{d} t} \frac{\mathrm{d}}{\mathrm{d} s}\right|_{t=0, s=0}\left(\beta e^{s A \beta^{-1}} e^{t B \beta^{-1}} e^{-s A \beta^{-1}} e^{-t B \beta^{-1}}\right) \\
& \quad=\beta A \beta^{-1} B \beta^{-1}-\beta B \beta^{-1} A \beta^{-1}=[A, B]_{\beta},
\end{aligned}
$$

which implies that (5.2) hold. 


\section{Integration of the derivation Hom-Lie algebra}

Let the triple $\left(\mathfrak{g},[\cdot, \cdot]_{\mathfrak{g}}, \phi_{\mathfrak{g}}\right)$ be a Hom-Lie algebra. In this section, we consider the derivation of $\left(\mathfrak{g},[\cdot, \cdot]_{\mathfrak{g}}, \phi_{\mathfrak{g}}\right)$ and show that it is the Hom-Lie algebra of the Hom-Lie group of automorphisms of $\left(\mathfrak{g},[\cdot, \cdot]_{\mathfrak{g}}, \phi_{\mathfrak{g}}\right)$.

Let us recall from Definition 2.6, the space of derivations of a Hom-Lie algebra $\left(\mathfrak{g},[\cdot, \cdot]_{\mathfrak{g}}, \phi_{\mathfrak{g}}\right)$ that is denoted by $\operatorname{Der}(\mathfrak{g})$. Then, we have the following proposition.

Proposition 6.1. Let $\left(\mathfrak{g},[\cdot, \cdot]_{\mathfrak{g}}, \phi_{\mathfrak{g}}\right)$ be a Hom-Lie algebra. Then $\left(\operatorname{Der}(\mathfrak{g}),[\cdot, \cdot]_{\phi_{\mathfrak{g}}}, \operatorname{Ad}_{\phi_{\mathfrak{g}}}\right)$ is a HomLie algebra, which is a subalgebra of the Hom-Lie algebra $\left(\mathfrak{g l}(\mathfrak{g}),[\cdot, \cdot]_{\phi_{\mathfrak{g}}}, \operatorname{Ad}_{\phi_{\mathfrak{g}}}\right)$ given by Proposition 2.8 .

Proof. The proof is a straightforward verification, and we leave the details to readers.

Proposition 6.2. A linear map $D: \mathfrak{g} \rightarrow \mathfrak{g}$ is a derivation of the Hom-Lie algebra $\left(\mathfrak{g},[\cdot, \cdot]_{\mathfrak{g}}, \phi_{\mathfrak{g}}\right)$ if and only if the map $D \circ \phi_{\mathfrak{g}}^{-1}: \mathfrak{g} \rightarrow \mathfrak{g}$ is a derivation of the Lie algebra $\left(\mathfrak{g},[\cdot, \cdot]_{\text {Lie }}\right)$ given in Lemma 2.2 .

Proof. Let us assume that $D: \mathfrak{g} \rightarrow \mathfrak{g}$ is a derivation of the Hom-Lie algebra $\left(\mathfrak{g},[\cdot, \cdot]_{\mathfrak{g}}, \phi_{\mathfrak{g}}\right)$, i.e.,

$$
D[x, y]_{\mathfrak{g}}=\left[\phi_{\mathfrak{g}}(x),\left(\operatorname{Ad}_{\phi_{\mathfrak{g}}^{-1}} D\right)(y)\right]_{\mathfrak{g}}+\left[\left(\operatorname{Ad}_{\phi_{\mathfrak{g}}^{-1}} D\right)(x), \phi(y)\right]_{\mathfrak{g}}, \quad \forall x, y \in \mathfrak{g} .
$$

We need to show that $D \circ \phi_{\mathfrak{g}}^{-1}: \mathfrak{g} \rightarrow \mathfrak{g}$ is a derivation of the Lie algebra $\left(\mathfrak{g},[\cdot, \cdot]_{\text {Lie }}\right)$. Equivalently, we need to show the following identity:

$$
D \circ \phi_{\mathfrak{g}}^{-1}[x, y]_{\mathrm{Lie}}=\left[D\left(\phi_{\mathfrak{g}}^{-1}(x)\right), y\right]_{\mathrm{Lie}}+\left[x, D\left(\phi_{\mathfrak{g}}^{-1}(y)\right)\right]_{\mathrm{Lie}}, \quad \forall x, y \in \mathfrak{g} .
$$

The left hand side of (6.1) can be written as

$$
\begin{aligned}
D \circ \phi_{\mathfrak{g}}^{-1}[x, y]_{\text {Lie }} & =D\left[\phi_{\mathfrak{g}}^{-2}(x), \phi_{\mathfrak{g}}^{-2}(y)\right]_{\mathfrak{g}} \\
& =\left[\phi_{\mathfrak{g}}^{-1}(x), \phi_{\mathfrak{g}}^{-1} \circ D \circ \phi_{\mathfrak{g}}^{-1}(y)\right]_{\mathfrak{g}}+\left[\phi_{\mathfrak{g}}^{-1} \circ D \circ \phi_{\mathfrak{g}}^{-1}(x), \phi_{\mathfrak{g}}^{-1}(y)\right]_{\mathfrak{g}},
\end{aligned}
$$

and the right hand side of (6.1) can be written as

$$
\begin{aligned}
& {\left[D\left(\phi_{\mathfrak{g}}^{-1}(x)\right), y\right]_{\mathrm{Lie}}+\left[x, D\left(\phi_{\mathfrak{g}}^{-1}(y)\right)\right]_{\mathrm{Lie}}} \\
& \quad=\left[\phi_{\mathfrak{g}}^{-1} \circ D \circ \phi_{\mathfrak{g}}^{-1}(x), \phi_{\mathfrak{g}}^{-1}(y)\right]_{\mathfrak{g}}+\left[\phi_{\mathfrak{g}}^{-1}(x), \phi_{\mathfrak{g}}^{-1} \circ D \circ \phi_{\mathfrak{g}}^{-1}(y)\right]_{\mathfrak{g}} .
\end{aligned}
$$

Thus, it implies that $(6.1)$ holds, i.e., $D \circ \phi_{\mathfrak{g}}^{-1}$ is a derivation of the Lie algebra $\left(\mathfrak{g},[\cdot, \cdot]_{\text {Lie }}\right)$.

Conversely, if $D \circ \phi_{\mathfrak{g}}^{-1}$ is a derivation of the Lie algebra $\left(\mathfrak{g},[\cdot, \cdot]_{\text {Lie }}\right)$, then it easily follows that $D$ is a derivation of the Hom-Lie algebra $\left(\mathfrak{g},[\cdot, \cdot]_{\mathfrak{g}}, \phi_{\mathfrak{g}}\right)$.

Definition 6.3. Let $\left(\mathfrak{g},[\cdot, \cdot]_{\mathfrak{g}}, \phi_{\mathfrak{g}}\right)$ be a Hom-Lie algebra. An automorphism of $\left(\mathfrak{g},[\cdot, \cdot]_{\mathfrak{g}}, \phi_{\mathfrak{g}}\right)$ is a map $\theta \in \mathrm{GL}(\mathfrak{g})$ such that

$$
\theta[x, y]_{\mathfrak{g}}=\left[\operatorname{Ad}_{\phi_{\mathfrak{g}}^{-1}} \theta(x), \operatorname{Ad}_{\phi_{\mathfrak{g}}^{-1}} \theta(y)\right]_{\mathfrak{g}}, \quad \forall x, y \in \mathfrak{g} .
$$

We denote the set of automorphisms of a Hom-Lie algebra $\left(\mathfrak{g},[\cdot, \cdot]_{\mathfrak{g}}, \phi_{\mathfrak{g}}\right)$ by Aut $(\mathfrak{g})$. In fact, there is a Hom-Lie group structure on the set Aut $(\mathfrak{g})$.

Proposition 6.4. Let $\left(\mathfrak{g},[\cdot, \cdot]_{\mathfrak{g}}, \phi_{\mathfrak{g}}\right)$ be a Hom-Lie algebra. Then the tuple (Aut $\left.(\mathfrak{g}), \diamond, \phi_{\mathfrak{g}}, \operatorname{Ad}_{\phi_{\mathfrak{g}}}\right)$ is a Hom-Lie subgroup of the Hom-Lie group $\left(\mathrm{GL}(\mathfrak{g}), \diamond, \phi_{\mathfrak{g}}, \operatorname{Ad}_{\phi_{\mathfrak{g}}}\right)$. 
Proof. First, we show that the structure map $\phi_{\mathfrak{g}}$ is an automorphism of the Hom-Lie algebra $\left(\mathfrak{g},[\cdot, \cdot]_{\mathfrak{g}}, \phi_{\mathfrak{g}}\right)$. It follows from the following expression:

$$
\phi_{\mathfrak{g}}[x, y]_{\mathfrak{g}}=\left[\phi_{\mathfrak{g}}(x), \phi_{\mathfrak{g}}(y)\right]_{\mathfrak{g}}=\left[\operatorname{Ad}_{\phi_{\mathfrak{g}}^{-1}} \phi_{\mathfrak{g}}(x), \operatorname{Ad}_{\phi_{\mathfrak{g}}^{-1}} \phi_{\mathfrak{g}}(y)\right]_{\mathfrak{g}}
$$

for all $x, y \in \mathfrak{g}$. Now, let $\theta \in \operatorname{Aut}(\mathfrak{g})$, then we have

$$
\theta\left[\operatorname{Ad}_{\phi_{\mathfrak{g}}^{-1}} \theta^{-1}(x), \operatorname{Ad}_{\phi_{\mathfrak{g}}^{-1}} \theta^{-1}(y)\right]_{\mathfrak{g}}=[x, y]_{\mathfrak{g}},
$$

which implies that $\theta^{-1}[x, y]_{\mathfrak{g}}=\left[\operatorname{Ad}_{\phi_{\mathfrak{g}}^{-1}} \theta^{-1}(x), \operatorname{Ad}_{\phi_{\mathfrak{g}}^{-1}} \theta^{-1}(y)\right]_{\mathfrak{g}}$ for all $x, y \in \mathfrak{g}$. Thus $\theta^{-1} \in \operatorname{Aut}(\mathfrak{g})$.

Moreover,

$$
\begin{aligned}
\theta_{1} \diamond \theta_{2}[x, y]_{\mathfrak{g}} & =\phi_{\mathfrak{g}} \circ \theta_{1} \circ \phi_{\mathfrak{g}}^{-1} \circ \theta_{2} \circ \phi_{\mathfrak{g}}^{-1}[x, y]_{\mathfrak{g}}=\phi_{\mathfrak{g}} \circ \theta_{1} \circ \phi_{\mathfrak{g}}^{-1}\left[\phi_{\mathfrak{g}}^{-1} \circ \theta_{2}(x), \phi_{\mathfrak{g}}^{-1} \circ \theta_{2}(y)\right]_{\mathfrak{g}} \\
& =\left[\theta_{1} \circ \phi_{\mathfrak{g}}^{-1} \circ \theta_{2}(x), \theta_{1} \circ \phi_{\mathfrak{g}}^{-1} \circ \theta_{2} \circ \phi_{\mathfrak{g}}(y)\right]_{\mathfrak{g}} \\
& =\left[\operatorname{Ad}_{\phi_{\mathfrak{g}}^{-1}}\left(\theta_{1} \diamond \theta_{2}\right)(x), \operatorname{Ad}_{\phi_{\mathfrak{g}}^{-1}}\left(\theta_{1} \diamond \theta_{2}\right)(y)\right]_{\mathfrak{g}},
\end{aligned}
$$

for all $\theta_{1}, \theta_{2} \in \operatorname{Aut}(\mathfrak{g})$, and $x, y \in \mathfrak{g}$. Therefore, $\theta_{1} \diamond \theta_{2} \in \operatorname{Aut}(\mathfrak{g})$. Finally, we have

$$
\operatorname{Ad}_{\phi_{\mathfrak{g}}} \theta[x, y]_{\mathfrak{g}}=\phi_{\mathfrak{g}} \circ \theta \circ \phi_{\mathfrak{g}}^{-1}[x, y]_{\mathfrak{g}}=[\theta(x), \theta(y)]_{\mathfrak{g}}=\left[\operatorname{Ad}_{\phi_{\mathfrak{g}}^{-1}}\left(\operatorname{Ad}_{\phi_{\mathfrak{g}}} \theta\right)(x), \operatorname{Ad}_{\phi_{\mathfrak{g}}^{-1}}\left(\operatorname{Ad}_{\phi_{\mathfrak{g}}} \theta\right)(y)\right]_{\mathfrak{g}},
$$

which implies that $\operatorname{Ad}_{\phi_{\mathfrak{g}}} \theta \in \operatorname{Aut}(\mathfrak{g})$. Hence, $\left(\operatorname{Aut}(\mathfrak{g}), \diamond, \phi_{\mathfrak{g}}, \operatorname{Ad}_{\phi_{\mathfrak{g}}}\right)$ is a Hom-Lie subgroup of the Hom-Lie group $\left(\mathrm{GL}(\mathfrak{g}), \diamond, \phi_{\mathfrak{g}}, \operatorname{Ad}_{\phi_{\mathfrak{g}}}\right)$.

Theorem 6.5. With the above notations, the triple $\left(\operatorname{Der}(\mathfrak{g}),[\cdot, \cdot]_{\phi_{\mathfrak{g}}}, A d_{\phi_{\mathfrak{g}}}\right)$ is the Hom-Lie algebra of the Hom-Lie group $\left(\operatorname{Aut}(\mathfrak{g}), \diamond, \phi_{\mathfrak{g}}, \operatorname{Ad}_{\phi_{\mathfrak{g}}}\right)$.

Proof. Let us first assume that $D$ is a derivation of the Hom-Lie algebra $\left(\mathfrak{g},[\cdot, \cdot]_{\mathfrak{g}}, \phi_{\mathfrak{g}}\right)$. By $(3.11)$, we have $\operatorname{Hexp}(t D)=\phi_{\mathfrak{g}} e^{t D \phi_{\mathfrak{g}}^{-1}}$. Then we prove that $\operatorname{Hexp}(t D)$ is an automorphism of the Hom-Lie algebra $\left(\mathfrak{g},[\cdot, \cdot]_{\mathfrak{g}}, \phi_{\mathfrak{g}}\right)$. For all $x, y \in \mathfrak{g}$, we get the following equation:

$$
\operatorname{Hexp}(t D)[x, y]_{\mathfrak{g}}=\operatorname{Hexp}(t D)\left[\phi_{\mathfrak{g}}(x), \phi_{\mathfrak{g}}(y)\right]_{\text {Lie }}=\phi_{\mathfrak{g}} e^{t D \phi_{\mathfrak{g}}^{-1}}\left[\phi_{\mathfrak{g}}(x), \phi_{\mathfrak{g}}(y)\right]_{\text {Lie }} .
$$

By Proposition 6.2, the map $D \circ \phi_{\mathfrak{g}}^{-1}: \mathfrak{g} \rightarrow \mathfrak{g}$ is a derivation of Lie algebra $\left(\mathfrak{g},[\cdot, \cdot]_{\text {Lie }}\right)$. This, in turn, implies that $e^{t D \phi_{\mathfrak{g}}^{-1}}$ is an automorphism of $\left(\mathfrak{g},[\cdot, \cdot]_{\text {Lie }}\right)$. Thus,

$$
\phi_{\mathfrak{g}} e^{t D \phi_{\mathfrak{g}}^{-1}}\left[\phi_{\mathfrak{g}}(x), \phi_{\mathfrak{g}}(y)\right]_{\text {Lie }}=\phi_{\mathfrak{g}}\left[e^{t D \phi_{\mathfrak{g}}^{-1}} \phi_{\mathfrak{g}}(x), e^{t D \phi_{\mathfrak{g}}^{-1}} \phi_{\mathfrak{g}}(y)\right]_{\text {Lie }}
$$

By Lemma 2.2, we have

$$
\begin{aligned}
\phi_{\mathfrak{g}}\left[e^{t D \phi_{\mathfrak{g}}^{-1}} \phi_{\mathfrak{g}}(x), e^{t D \phi_{\mathfrak{g}}^{-1}} \phi_{\mathfrak{g}}(y)\right]_{\text {Lie }} & =\phi_{\mathfrak{g}}\left[\phi_{\mathfrak{g}}^{-1} e^{t D \phi_{\mathfrak{g}}^{-1}} \phi_{\mathfrak{g}}(x), \phi_{\mathfrak{g}}^{-1} e^{t D \phi_{\mathfrak{g}}^{-1}} \phi_{\mathfrak{g}}(y)\right]_{\mathfrak{g}} \\
& =\left[e^{t D \phi_{\mathfrak{g}}^{-1}} \phi_{\mathfrak{g}}(x), e^{t D \phi_{\mathfrak{g}}^{-1}} \phi_{\mathfrak{g}}(y)\right]_{\mathfrak{g}}
\end{aligned}
$$

i.e.,

$$
\left[e^{t D \phi_{\mathfrak{g}}^{-1}} \phi_{\mathfrak{g}}(x), e^{t D \phi_{\mathfrak{g}}^{-1}} \phi_{\mathfrak{g}}(y)\right]_{\mathfrak{g}}=\left[\operatorname{Ad}_{\phi_{\mathfrak{g}}{ }^{-1}} \operatorname{Hexp}(t D)(x), \operatorname{Ad}_{\phi_{\mathfrak{g}}^{-1}} \operatorname{Hexp}(t D)(y)\right]_{\mathfrak{g}} .
$$

Therefore,

$$
\operatorname{Hexp}(t D)[x, y]_{\mathfrak{g}}=\left[\operatorname{Ad}_{\phi_{\mathfrak{g}}{ }^{-1}} \operatorname{Hexp}(t D)(x), \operatorname{Ad}_{\phi_{\mathfrak{g}}{ }^{1}} \operatorname{Hexp}(t D)(y)\right]_{\mathfrak{g}} .
$$

Conversely, we show that if $D: \mathfrak{g} \rightarrow \mathfrak{g}$ is a linear map and it satisfies (6.4), then $D$ is a derivation of the Hom-Lie algebra $\left(\mathfrak{g},[\cdot, \cdot]_{\mathfrak{g}}, \phi_{\mathfrak{g}}\right)$. Note that (6.2) and (6.3) implies that

$$
\phi_{\mathfrak{g}} e^{t D \phi_{\mathfrak{g}}^{-1}}\left[\phi_{\mathfrak{g}}(x), \phi_{\mathfrak{g}}(y)\right]_{\text {Lie }}=\left[e^{t D \phi_{\mathfrak{g}}^{-1}} \phi_{\mathfrak{g}}(x), e^{t D \phi_{\mathfrak{g}}^{-1}} \phi_{\mathfrak{g}}(y)\right]_{\mathfrak{g}}=\phi_{\mathfrak{g}}\left[e^{t D \phi_{\mathfrak{g}}^{-1}} \phi_{\mathfrak{g}}(x), e^{t D \phi_{\mathfrak{g}}^{-1}} \phi_{\mathfrak{g}}(y)\right]_{\text {Lie }}
$$

for all $x, y \in \mathfrak{g}$. Therefore, $D \phi_{\mathfrak{g}}^{-1}$ is a derivation of the Lie algebra $\left(\mathfrak{g},[\cdot, \cdot]_{\text {Lie }}\right)$. Subsequently, from Proposition 6.2, it follows that $D$ is a derivation of the Hom-Lie algebra $\left(\mathfrak{g},[\cdot, \cdot]_{\mathfrak{g}}, \phi_{\mathfrak{g}}\right)$. 
Remark 6.6. Consider the Hom-Lie algebra $\left({ }_{q} \mathfrak{s l}_{2},[\cdot, \cdot], \alpha\right)$ given in $[12$, Example 1], where

$$
[e, f]=\frac{1+q}{2} h, \quad[h, e]=2 e, \quad[h, f]=-2 q f
$$

and

$$
\alpha(e)=\frac{q^{-1}+1}{2} e, \quad \alpha(h)=h, \quad \alpha(f)=\frac{q+1}{2} f .
$$

Here $\{e, f, h\}$ is the basis of $\mathfrak{s l}_{2}$. It is straightforward to deduce that

$$
\alpha([e, f])=\frac{q+1}{2} h, \quad[\alpha(e), \alpha(f)]=\frac{q^{-1}+1}{2} \frac{q+1}{2} \frac{q+1}{2} h,
$$

which implies that $\alpha$ does not preserve $[\cdot, \cdot]$, i.e., $\alpha$ is not an algebraic morphism. So the HomLie algebra ${ }_{q} \mathfrak{s l}_{2}$ is not a multiplicative Hom-Lie algebra. Thus the integration-differentiation approach developed in this paper does not apply to this concrete example. We will study the integration of this more general case in the future.

\section{Acknowledgements}

We give our warmest thanks to the referees for very helpful suggestions that improve the paper. Research supported by NSFC (11922110)

\section{References}

[1] Ammar F., Ejbehi Z., Makhlouf A., Cohomology and deformations of Hom-algebras, J. Lie Theory 21 (2011), 813-836, arXiv:1005.0456.

[2] Bohm G., Vercruysse J., BiHom Hopf algebras viewed as Hopf monoids, arXiv:2003.08819.

[3] Caenepeel S., Goyvaerts I., Monoidal Hom-Hopf algebras, Comm. Algebra 39 (2011), 2216-2240, arXiv:0907.0187.

[4] Cai L., Liu J., Sheng Y., Hom-Lie algebroids, Hom-Lie bialgebroids and Hom-Courant algebroids, J. Geom. Phys. 121 (2017), 15-32, arXiv:1605.04752.

[5] Cai L., Sheng Y., Hom-big brackets: theory and applications, SIGMA 12 (2016), 014, 18 pages, arXiv:1507.04061.

[6] Hartwig J.T., Larsson D., Silvestrov S.D., Deformations of Lie algebras using $\sigma$-derivations, J. Algebra 295 (2006), 314-361, arXiv:math.QA/0408064.

[7] Hassanzadeh M., Hom-groups, representations and homological algebra, Colloq. Math. 158 (2019), 21-38, arXiv:1801.07398.

[8] Hassanzadeh M., Lagrange's theorem for Hom-groups, Rocky Mountain J. Math. 49 (2019), 773-787, arXiv:1803.07678.

[9] Hu N., $q$-Witt algebras, $q$-Lie algebras, $q$-holomorph structure and representations, Algebra Colloq. 6 (1999), 51-70, arXiv:math.QA/0512526.

[10] Larsson D., Silvestrov S.D., Quasi-hom-Lie algebras, central extensions and 2-cocycle-like identities, J. Algebra 288 (2005), 321-344, arXiv:math.RA/0408061.

[11] Larsson D., Silvestrov S.D., Quasi-Lie algebras, in Noncommutative Geometry and Representation Theory in Mathematical Physics,Contemp. Math., Vol. 391, Amer. Math. Soc., Providence, RI, 2005, 241-248.

[12] Larsson D., Silvestrov S.D., Quasi-deformations of $\mathfrak{s l}_{2}(\mathbb{F})$ using twisted derivations, Comm. Algebra 35 (2007), 4303-4318, arXiv:math.RA/0506172.

[13] Laurent-Gengoux C., Makhlouf A., Teles J., Universal algebra of a Hom-Lie algebra and group-like elements, J. Pure Appl. Algebra 222 (2018), 1139-1163, arXiv:1505.02439.

[14] Laurent-Gengoux C., Teles J., Hom-Lie algebroids, J. Geom. Phys. 68 (2013), 69-75, arXiv:1211.2263. 
[15] Makhlouf A., Silvestrov S., Notes on 1-parameter formal deformations of Hom-associative and Hom-Lie algebras, Forum Math. 22 (2010), 715-739, arXiv:0712.3130.

[16] Mandal A., Mishra S.K., Hom-Lie-Rinehart algebras, Comm. Algebra 46 (2018), 3722-3744, arXiv:1610.01477.

[17] Sheng Y., Representations of hom-Lie algebras, Algebr. Represent. Theory 15 (2012), 1081-1098, arXiv:1005.0140.

[18] Sheng Y., Chen D., Hom-Lie 2-algebras, J. Algebra 376 (2013), 174-195, arXiv:1110.3405.

[19] Sheng Y., Xiong Z., On Hom-Lie algebras, Linear Multilinear Algebra 63 (2015), 2379-2395, arXiv:1411.6839.

[20] Yau D., Enveloping algebras of Hom-Lie algebras, J. Gen. Lie Theory Appl. 2 (2008), 95-108, arXiv:0709.0849.

[21] Yau D., Hom-quantum groups: I. Quasi-triangular Hom-bialgebras, J. Phys. A: Math. Theor. 45 (2012), 065203, 23 pages, arXiv:0906.4128. 